\title{
IRF5 governs liver macrophage activation that promotes hepatic fibrosis in mice and humans
}

Fawaz Alzaid, ${ }^{1}$ Floriane Lagadec, ${ }^{2}$ Miguel Albuquerque, ${ }^{2}$ Raphaëlle Ballaire, ${ }^{1}$ Lucie Orliaguet, ${ }^{1}$ Isabelle Hainault, ${ }^{1}$ Corinne Blugeon, ${ }^{3}$ Sophie Lemoine, ${ }^{3}$ Agnès Lehuen, ${ }^{4}$ David G. Saliba, ${ }^{5}$ Irina A. Udalova, ${ }^{5}$ Valérie Paradis, ${ }^{2}$ Fabienne Foufelle, ${ }^{1}$ and Nicolas Venteclef'

'Institut National de la Santé et de la Recherche Médicale (INSERM) UMRS 1138, Sorbonne Universités, UPMC Université Paris 06; Sorbonne Paris Cité, Université Paris Descartes, Université Paris Diderot; and Centre de Recherche des Cordeliers, Paris, France. ${ }^{2}$ INSERM UMRS 1149 and Department of Pathology Beaujon Hospital, Assistance Publique-Hôpitaux de Paris, Clichy, France. ${ }^{3}$ École normale supérieure, PSL Research University, Centre National de la Recherche Scientifique (CNRS), INSERM, Institut de Biologie de l'École Normale Supérieure (IBENS), Plateforme Génomique, Paris, France. ${ }^{4}$ INSERM UMRS 1016, Institut Cochin, Paris, France; CNRS UMR_S 8104, Paris, France; and Laboratoire d'Excellence INFLAMEX, Université Paris Descartes, Sorbonne Paris Cité, Paris, France. ${ }^{5}$ Kennedy Institute of Rheumatology, University of Oxford, Oxford, United Kingdom.

Hepatic fibrosis arises from inflammation in the liver initiated by resident macrophage activation and massive leukocyte accumulation. Hepatic macrophages hold a central position in maintaining homeostasis in the liver and in the pathogenesis of acute and chronic liver injury linked to fibrogenesis. Interferon regulatory factor 5 (IRF5) has recently emerged as an important proinflammatory transcription factor involved in macrophage activation under acute and chronic inflammation. Here, we revealed that IRF5 is significantly induced in liver macrophages from human subjects developing liver fibrosis from nonalcoholic fatty liver disease or hepatitis $C$ virus infection. Furthermore, IRF5 expression positively correlated with clinical markers of liver damage, such as plasma transaminase and bilirubin levels. Interestingly, mice lacking IRF5 in myeloid cells (MKO) were protected from hepatic fibrosis induced by metabolic or toxic stresses. Transcriptional reprogramming of macrophages lacking IRF5 was characterized by immunosuppressive and antiapoptotic properties. Consequently, IRF5 MKO mice respond to hepatocellular stress by promoting hepatocyte survival, leading to complete protection from hepatic fibrogenesis. Our findings reveal a regulatory network, governed by IRF5, that mediates hepatocyte death and liver fibrosis in mice and humans. Therefore, modulating IRF5 function may be an attractive approach to experimental therapeutics in fibroinflammatory liver disease.

Conflict of interest: The authors have declared that no conflict of interest exists.

Submitted: June 10, 2016 Accepted: October 25, 2016 Published: December 8, 2016

Reference information: JCl Insight. 2016;1(20):e88689. doi:10.1172/jci.insight.88689.

\section{Introduction}

Chronic liver disease has multiple etiologies, including viral infection and metabolic disorders. Regardless of initial causes, progression to liver fibrosis and cirrhosis requires an inflammatory component $(1,2)$. The fibrogenic process is a continuous wound healing response mediated by hepatic stellate cell (HSC) activation and interaction between innate and adaptive immune cells (3). Excessive wound healing due to uncontrolled hepatocyte death upon persistent hepatocellular stress results in pathologic fibrogenesis (4). Evidence from clinical and animal studies suggests that hepatic macrophages play an important role in all stages of liver disease (5). An important property of macrophages in liver pathology is their ability to induce hepatocellular apoptosis $(6,7)$. During liver injury, resident macrophages, known as Kupffer cells (KCs) are critical in the initial stress response (4). KCs respond by rapidly producing cytokines, including IL1 $\beta$ and TNF $\alpha$. Inflammatory cytokine release results in leukocyte recruitment (2). Leukocyte-derived death receptor ligand release propagates hepatocyte apoptosis, contributing to hepatopathology $(1,8)$. These death effectors induce apoptosis through engaging TNF receptor superfamily members on hepatocytes (9). Resulting hepatocyte death is central to liver pathophysiology in common disease models $(7,9)$. Through this process, macrophages are known to participate in regulated hepatocyte death, contributing 
to the pathogenesis of nonalcoholic steatohepatitis (NASH) and viral hepatitis C (HCV) (4). Interestingly, targeted deletion of resident and infiltrating macrophages during fibrogenesis ameliorates the end phenotype, while macrophage depletion during resolution exacerbates fibrosis (6). These opposing actions are in part explained by variation in infiltrated macrophage subsets destined to play different roles in liver fibrosis $(6,10)$.

Macrophages are characterized by their high plasticity in response to their microenvironment. Traditionally, macrophages are categorized by their terminal differentiation stage: proinflammatory (M1), wound healing (M2), or immunosuppressive $\left(\mathrm{M}_{\text {reg }}\right)$. This macrophage differentiation is dependent on regulatory cascades controlled by interferon regulatory factors (IRFs) (11). IRFs are a family of transcription factors involved in immunoregulation and immune cell differentiation through TLR signaling (12). We have recently documented that the fifth member of the IRFs, IRF5, is expressed in myeloid cells and responsible for M1 macrophage polarization (13-19). Through macrophage activation, IRF5 also elicits Th cell type 1 and 17 responses in acute and chronic inflammation $(14,18)$. More recently, we demonstrated the metabolic activation of IRF5 in obesity and its involvement in adipose tissue expansion. IRF5 knockout prevents adipose tissue expansion through alternative polarization of macrophage and $\mathrm{T}$ cell responses (16).

In the current study, we reveal increased expression of IRF5 in human liver fibrosis due to HCV infection and nonalcoholic fatty liver disease (NAFLD). Additionally, IRF5 expression correlates with markers of liver damage (bilirubin and transaminase), suggesting a key function in liver pathology. This causal association is confirmed in mice with a myeloid deficiency of IRF5 (IRF5 MKO). IRF5 MKO mice are resistant to liver fibrosis upon carbon tetrachloride-induced ( $\mathrm{CCl}_{4}$-induced) experimental fibrosis, under a NAFLD-like pathology induced by methionine and choline-deficient (MCD) feeding and due to cholestatic injury through bile duct ligation (BDL). Underlying this protective phenotype is an immunosuppressive polarization of IRF5-deficient liver macrophages. Consequently, hepatocytes are desensitized to death receptor stimulation, in turn ameliorating the initial response to hepatocellular stress.

\section{Results}

IRF5 expression is induced in human liver fibrosis. To address the association of IRF5 with the development of human liver fibrosis and steatohepatitis, we quantified mRNA levels of IRF5 in liver biopsies from subjects with normal liver, fatty liver, NASH, and HCV. Significantly higher expression of IRF5 was observed in NASH and HCV groups compared with controls (Figure 1A). In this cohort, the expression of IRF5 was strongly induced with fibrosis (stages F2-F4), but not with fatty liver (steatosis) (Figure 1B and Supplemental Figure 1A; supplemental material available online with this article; doi:10.1172/jci.insight.88689DS1).

To confirm gene expression results and the cell specificity of IRF5 expression, we analyzed, by IHC, the localization of IRF5 and CD68 (a pan-macrophage marker) in liver sections from the same subjects. IRF5 immunostaining was restricted to liver sinusoids and fibrous septa, and colocalized with CD68 immunostaining. Of note, we observed no localization with endothelial cells or HSCs (Supplemental Figure 1B). Furthermore, IRF5- and CD68-positive staining increased with the extent of liver damage and fibrosis in NASH and HCV (Figure 1C). Next, we investigated correlations between IRF5 mRNA levels and main liver function parameters. IRF5 expression correlated positively with plasma transaminases and bilirubin levels, and negatively with prothrombin time, indicating increased expression of IRF5 with markers reflecting liver damage (Figure 1D). These observations in humans strongly suggest that IRF5 is involved in the pathogenesis of steatohepatitis and liver fibrosis.

In order to further investigate the role of IRF5 in liver fibrosis, we used murine models of NASH and hepatic fibrosis. Chronic hepatocellular stress is induced by repeated administration of $\mathrm{CCl}_{4}$ for induction of experimental fibrosis; or MCD feeding or BDL for induction of a NASH-like pathology or cholestatic liver injury, respectively. Following treatments, fibrogenesis was assessed by red picrosirius (RP) staining, structural damage was assessed by $\mathrm{H} \& \mathrm{E}$ staining, and macrophage accumulation was quantified by $\mathrm{F} 4 / 80^{+}$ staining. We confirmed that MCD feeding leads to lipid accumulation, mild fibrosis, and macrophage accumulation; BDL leads to intermediate fibrosis, macrophage accumulation, and parenchymal necrosis, whereas $\mathrm{CCl}_{4}$ treatment leads to pronounced fibrosis and massive macrophage accumulation (Figure 2, $\mathrm{A}$ and $\mathrm{B}$ ). In mice treated with $\mathrm{MCD}, \mathrm{BDL}$, or $\mathrm{CCl}_{4}$, IRF5 expression was significantly increased relative to that in untreated mice, as measured by IHC analysis. Increased IRF5 expression was concordant with severity of liver damage and macrophage $\left(\mathrm{F} 4 / 80^{+}\right)$accumulation (Figure $\left.2 \mathrm{~B}\right)$. As we previously reported, expression of IRF5 was not modulated in liver or liver macrophages of mice fed a high-fat diet (16) (Sup- 
A

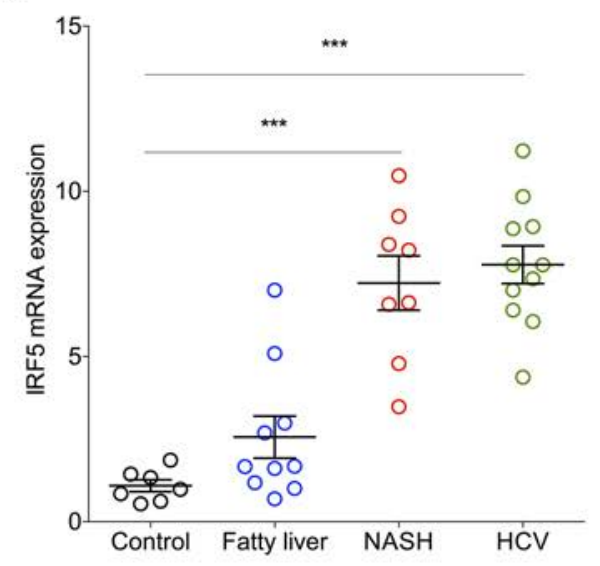

B

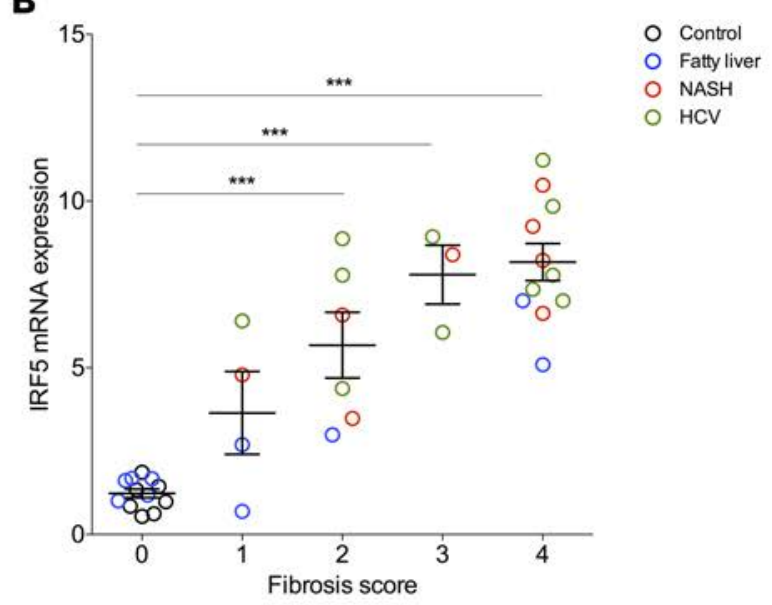

C Fatty liver

Fatty liver

$\mathrm{NASH}$

$\mathrm{HCV}$

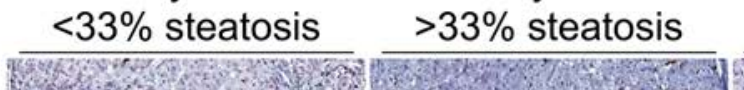

steatosis + fibrosis fibrosis
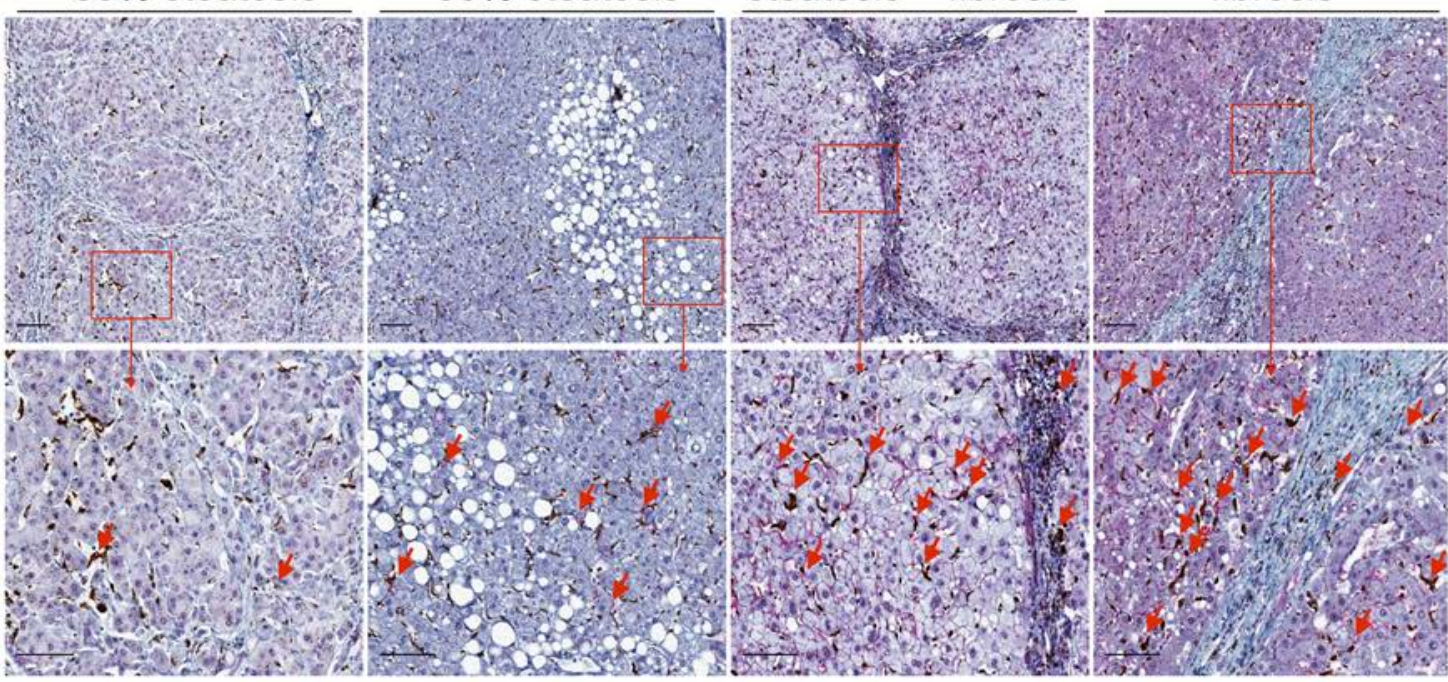
IRF5
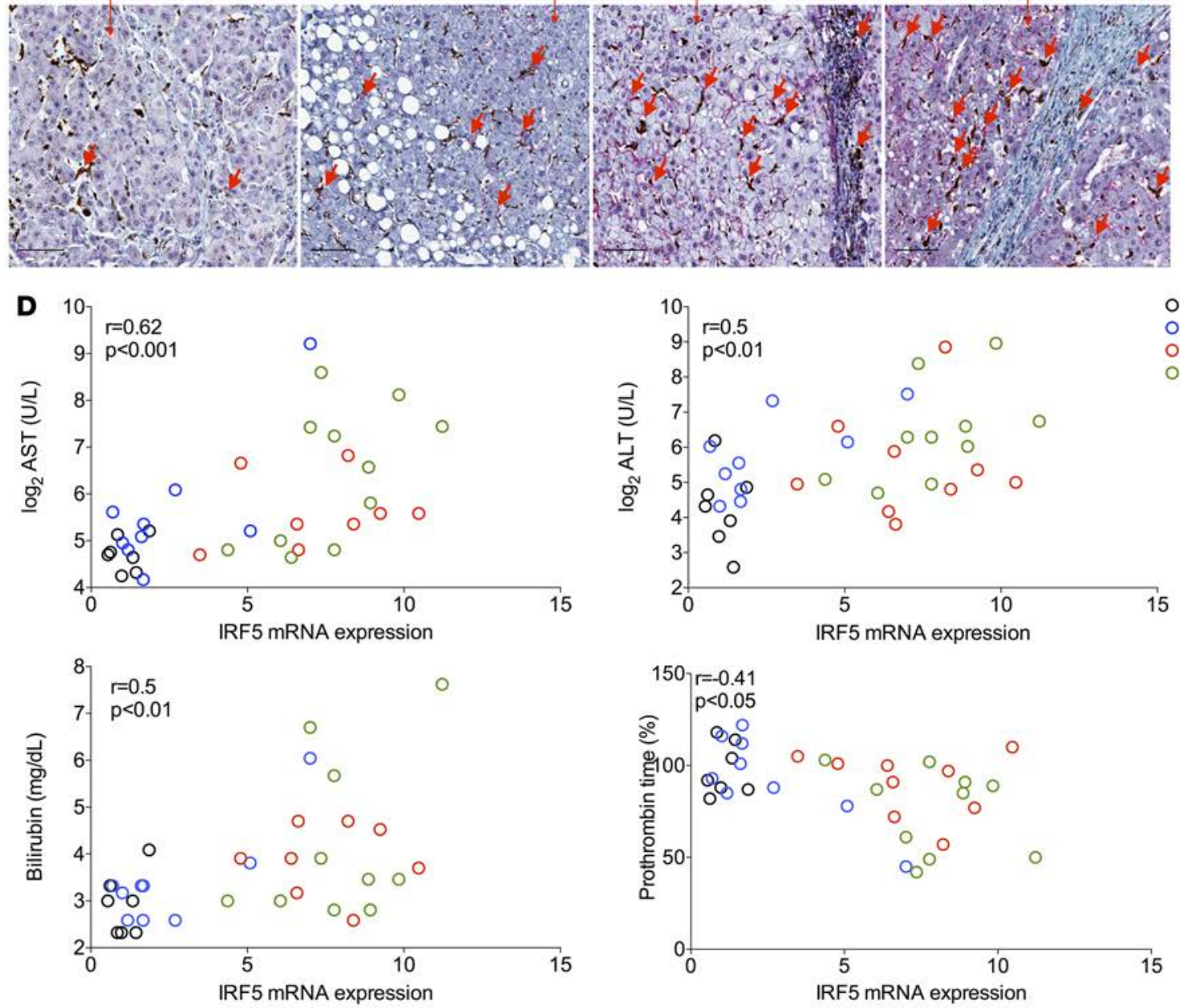
Figure 1. IRF5 is expressed in human hepatic macrophages and is induced in human NASH and hepatic fibrosis. (A) Interferon regulatory factor 5 (IRF5) mRNA expression in liver biopsies from control patients with normal liver $(n=7)$ and patients with fatty liver $(n=10)$, nonalcoholic steatohepatitis (NASH; $n=8$ ), and hepatitis C virus infection (HCV; $n=11)$. (B) IRF5 mRNA expression in the same cohort of patients $(n=36)$ stratified by fibrosis stage. (C) Representative images of IRF5 (pink stain) and CD68 (brown stain) immunostaining in liver sections from selected patients. Colocalization of stains shown by red arrows. (D) Correlative analyses of IRF5 mRNA expression with plasma aspartate and alanine transaminase (AST and ALT, respectively) levels, bilirubin levels, and prothrombin time from the same cohort of patients $(n=36)$. Scale bars: $100 \mu \mathrm{m}$. Differences between patient groups determined by 1-way ANOVA. Correlative analyses were assessed by Spearman's test. All values reported as mean \pm SEM. ${ }^{* *} P<0.001$.

plemental Figure 2, A and B). Interestingly, IRF5 ${ }^{+}$staining was localized to the same regions as $\mathrm{F} / 80^{+}$ staining, indicating localization of IRF5 expression to murine liver macrophages. Both IRF5 and F4/80 localized to sinusoidal regions, inflammatory infiltrates, and fibrous septa following $\mathrm{MCD}, \mathrm{BDL}$, or $\mathrm{CCl}_{4}$ treatment (Figure 2B). To confirm the induction of IRF5 in liver macrophages, mRNA levels of IRF5 were quantified by qRT-PCR in liver and in hepatic macrophages ( $\mathrm{F} 4 / 80^{+}$cells) from the same animals. IRF5 expression was induced in liver and liver macrophages following $\mathrm{MCD}, \mathrm{BDL}$, and $\mathrm{CCl}_{4}$ treatment (Figure 2C). Increased expression was specific to liver macrophages, while no modulation of IRF5 was seen in hepatocytes or F4/80- cells (Supplemental Figure 3, A and B). Furthermore, IRF5 expression in macrophages correlated with several markers of disease progression, namely macrophage accumulation (F4/80 expression), plasma transaminases, and fibrosis (RP staining and Col1 $\alpha 1$ expression) (Figure 2D).

Myeloid deficiency of IRF5 prevents hepatic fibrosis in vivo. To further address the causal association among IRF5, liver inflammation, and fibrosis, we generated MKO mice as previously described (16). Deletion of IRF5 expression in hepatic macrophages of MKO mice was confirmed by IHC, immunoblot and qRT-PCR (Figure 3A and Supplemental Figure 4A). We subjected MKO mice and wild-type littermates (MWT) to MCD feeding for 4 weeks, BDL for up to 10 days, and repeated $\mathrm{CCl}_{4}$ administration for 6 weeks; the resulting hepatic phenotype was analyzed. In all models, livers from MKO mice underwent significantly less structural change (H\&E staining) than those of MWT mice (Figure 3B). Indeed, quantification of fibrosis (RP staining) revealed decreased accumulation of collagen in $\mathrm{MKO}$ mice, accompanied by decreased Col1 11 mRNA expression compared with MWT upon MCD, BDL, and $\mathrm{CCl}_{4}$ treatment (Figure $3 \mathrm{C}$ ). HSC activation, a major process leading to collagen deposition (20), was also decreased in MKO mice in both models. This was evaluated by quantification of $\alpha$ SMA expression by IHC and qRT-PCR analyses (Figure 3D). The protected liver phenotype in MKO mice was reflected by decreased plasma transaminase levels in MKO mice relative to MWT mice in both models (Figure 3B). These results indicate a lower degree of liver damage and remodeling in $\mathrm{MKO}$ mice upon $\mathrm{MCD}, \mathrm{BDL}$, and $\mathrm{CCl}_{4}$ treatment.

Since IRF5 expression was restricted to hepatic F4/80 cells (Figure 2C and Supplemental Figure $3 \mathrm{~B})$, our next objective was to define the phenotype of the hepatic macrophage $\left(\mathrm{F} 4 / 80^{+}\right)$population in MWT and $\mathrm{MKO}$ mice following $\mathrm{MCD}, \mathrm{BDL}$, and $\mathrm{CCl}_{4}$ treatment. Interestingly, macrophage number was strongly reduced in liver from MKO mice compared with MWT mice under all treatments, as quantified by F4/80 immunostaining and gene expression in total liver (Figure 4A). Furthermore, FACS analyses of liver mononuclear cells (LMNCs) revealed decreased accumulation of $\mathrm{F} 4 / 80^{+} \mathrm{CD} 11 \mathrm{~b}^{+}$macrophages in liver from MKO mice (Figure 4B). Next, we identified the polarization of the hepatic macrophage population through further gating onto the proinflammatory M1 macrophage marker CD11c and the antiinflammatory $\mathrm{M} 2 / \mathrm{M}_{\mathrm{Reg}}$ macrophage marker CD206. We observed a phenotypic switch in the macrophage population, with a decrease in CD11 ${ }^{+}$macrophages and an increase in CD206 ${ }^{+}$macrophages in $\mathrm{MKO}$ relative to MWT mice under all treatments (Figure 4C). Our findings suggest that downregulation of the IRF5 pathway and subsequent antiinflammatory polarization of $\mathrm{MKO}$ hepatic macrophages is implicated in the hepatoprotective phenotype upon $\mathrm{MCD}, \mathrm{BDL}$, and $\mathrm{CCl}_{4}$ treatments

To identify the transcriptional pathways differentially expressed in liver macrophages, we carried out transcriptomic analyses by RNA sequencing on hepatic $\mathrm{F} 4 / 80^{+}$cells from MWT and MKO mice treated with $\mathrm{CCl}_{4}$. Transcriptome analyses revealed $\sim 1,300$ differentially expressed transcripts between $\mathrm{MKO}$ and MWT mice. Ontological clustering and pathway analyses revealed enrichment of multiple terms among downregulated transcripts regulating apoptosis, cytokine signaling, TLR signaling, and T cell activation (Tables 1 and 2). As expected, the TLRs/Myd88/IRF5 pathway was downregulated in hepatic F4/80 $0^{+}$cells from MKO mice (Figure 5A). Consequently, proinflammatory genes (IL1 $\beta$, IL6, MHC II, TNF) were downregulated, while antiinflammatory and immunosuppressive genes (TGF $\beta 1$, IL10, ARG1, CD206) were upregulated, corroborating the phenotypic switch seen with flow cytometric analyses. Differential expres- 
A
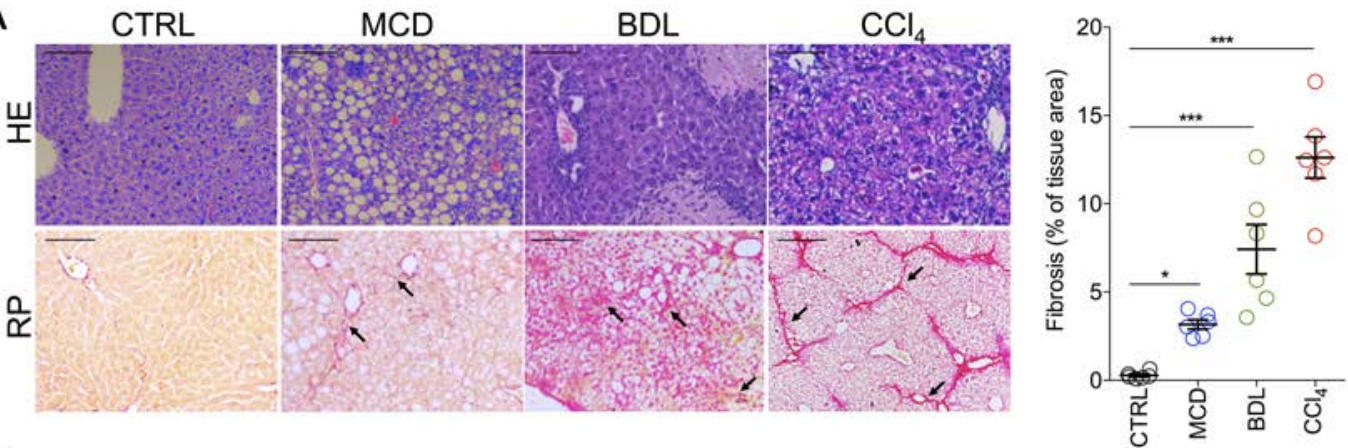

B CTRL MCD

BDL $\mathrm{CCl}_{4}$
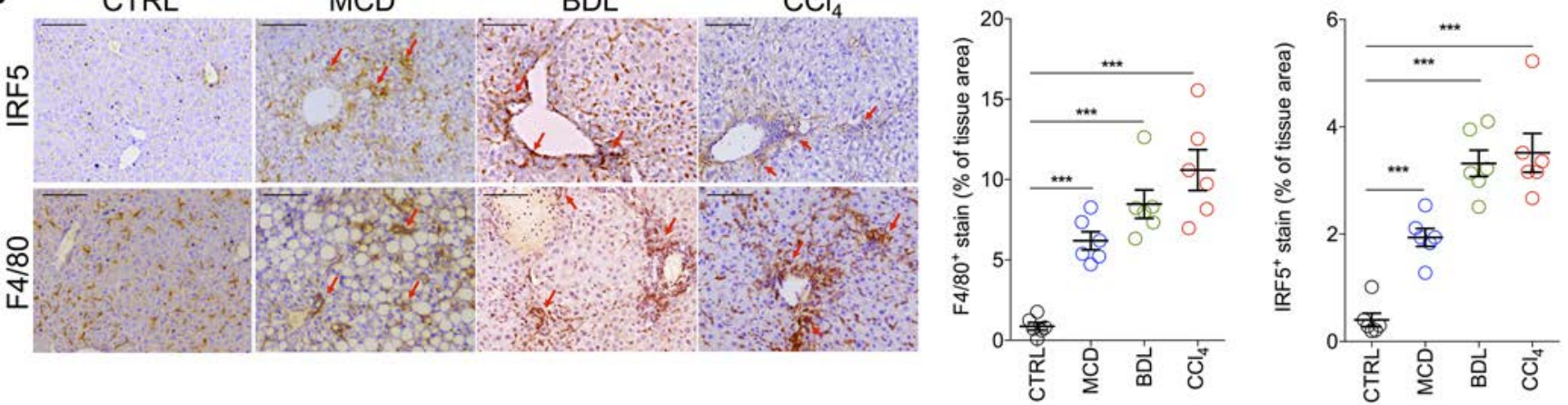

C
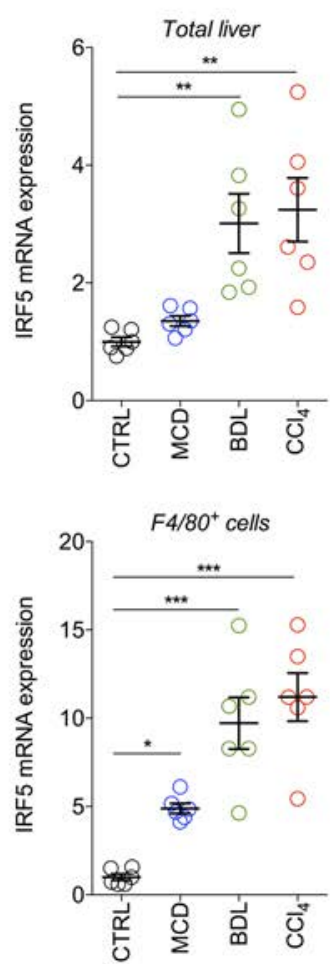

D
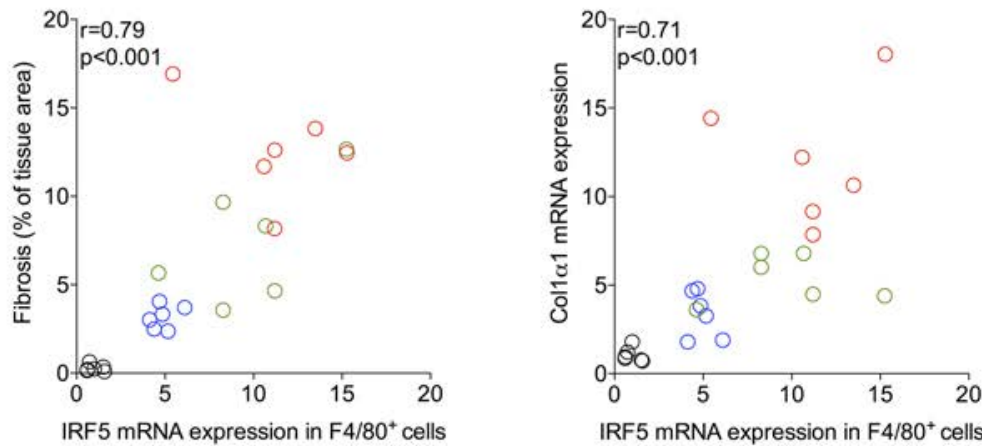

IRF5 mRNA expression in F4/80+ cells

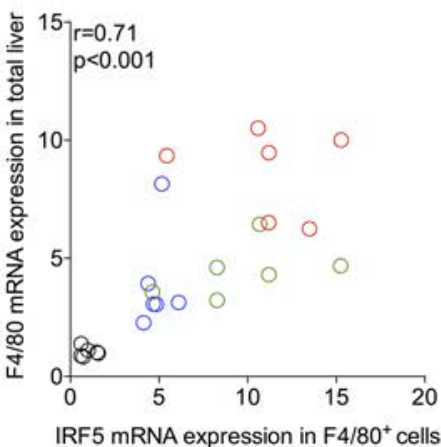

Figure 2. IRF5 is induced in mouse liver macrophages in NASH and experimental fibrosis models. Mice were maintained on a methionine and cholinedeficient (MCD) diet or underwent bile duct ligation (BDL) for induction of nonalcoholic steatohepatitis (NASH) or were treated with carbon tetrachloride $\left(\mathrm{CCI}_{4}\right)$ for induction of experimental fibrosis, and compared with mice with healthy liver maintained on a normal chow diet (CTRL). (A) Representative histological analysis of liver sections of mice maintained on normal chow, MCD, BDL, or CCl 4 , stained with $\mathrm{H} \& \mathrm{E}$ and red picrosirius (RP) to visualize fibrosis. Right panel: quantification of fibrosis. (B) Representative immunostaining of interferon regulatory factor 5 (IRF5) and F4/80 on liver sections. Right panels: quantification of immunostaining. (C) IRF5 mRNA expression in total liver lysate and hepatic $\mathrm{F} 4 / 80^{+}$cells of mice maintained on normal chow, $\mathrm{MCD}, \mathrm{BDL}$, or $\mathrm{CCl}$ (D) Correlative analyses of IRF5 mRNA expression in liver F4/80+ cells of mice maintained on normal chow, MCD, BDL, or $\mathrm{CCl}_{4}$. Correlations were determined for fibrosis, liver collagen type 1 alpha 1 (Col1 1 1) mRNA expression, plasma aspartate transferase (AST) and alanine transferase (ALT), and liver F4/80 mRNA

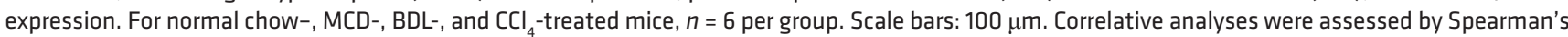
test. Differences between diets/treatments determined by 1-way ANOVA. All values reported as mean $\pm \mathrm{SEM}$. ${ }^{*} P<0.05,{ }^{* *} P<0.01,{ }^{* * *} P<0.001$. 
A

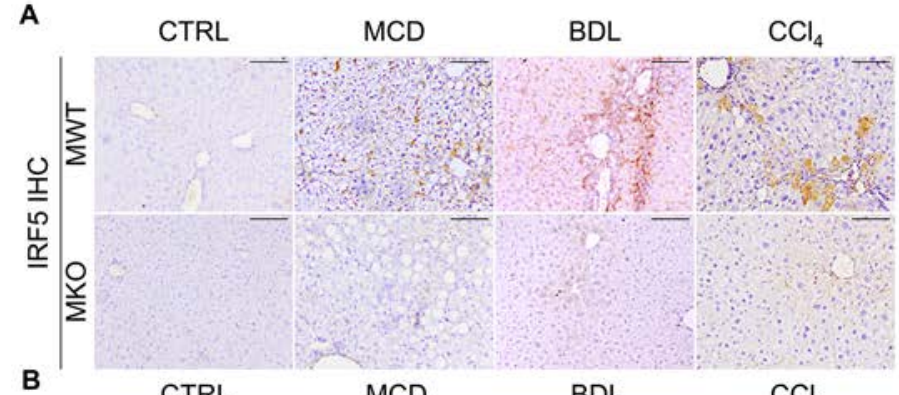

B
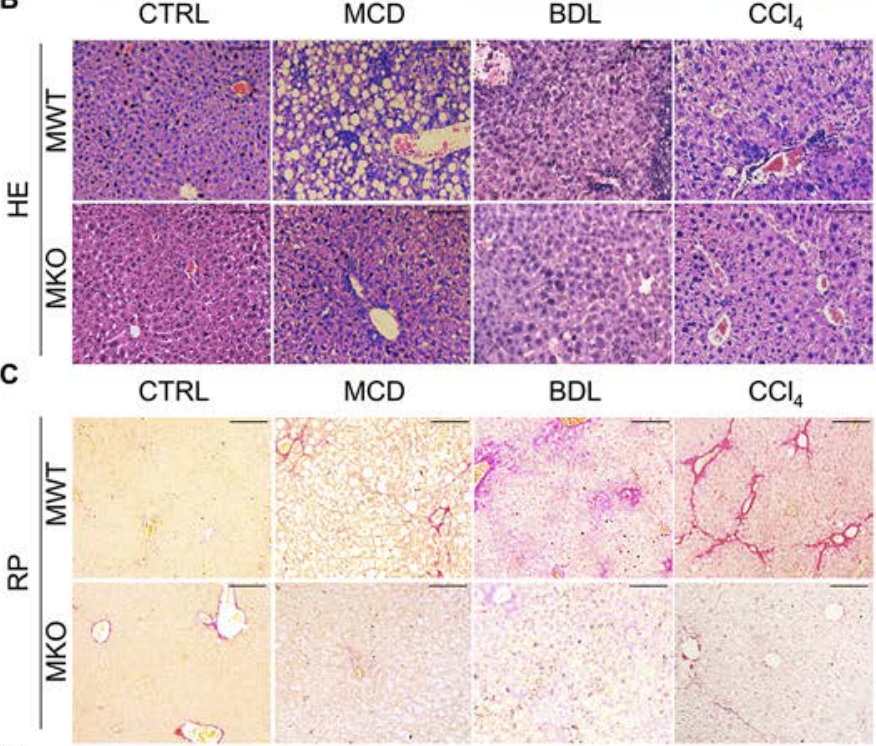

D

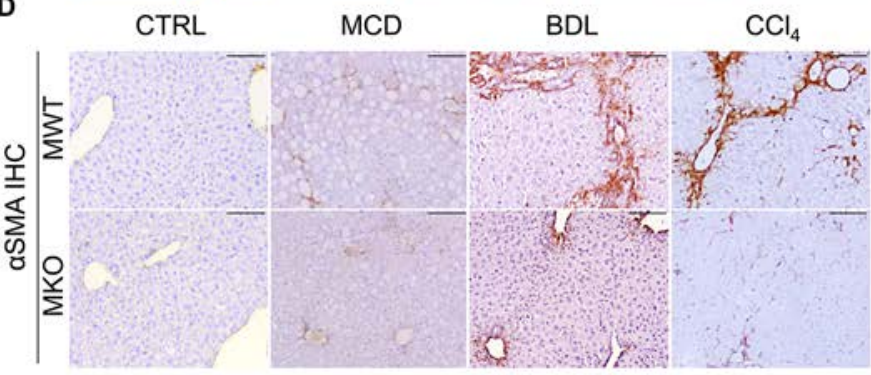

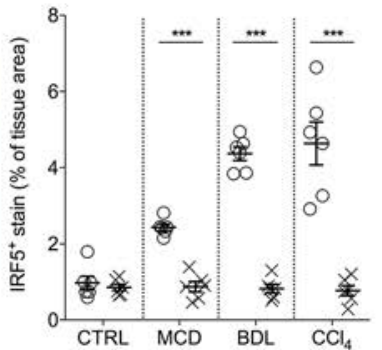
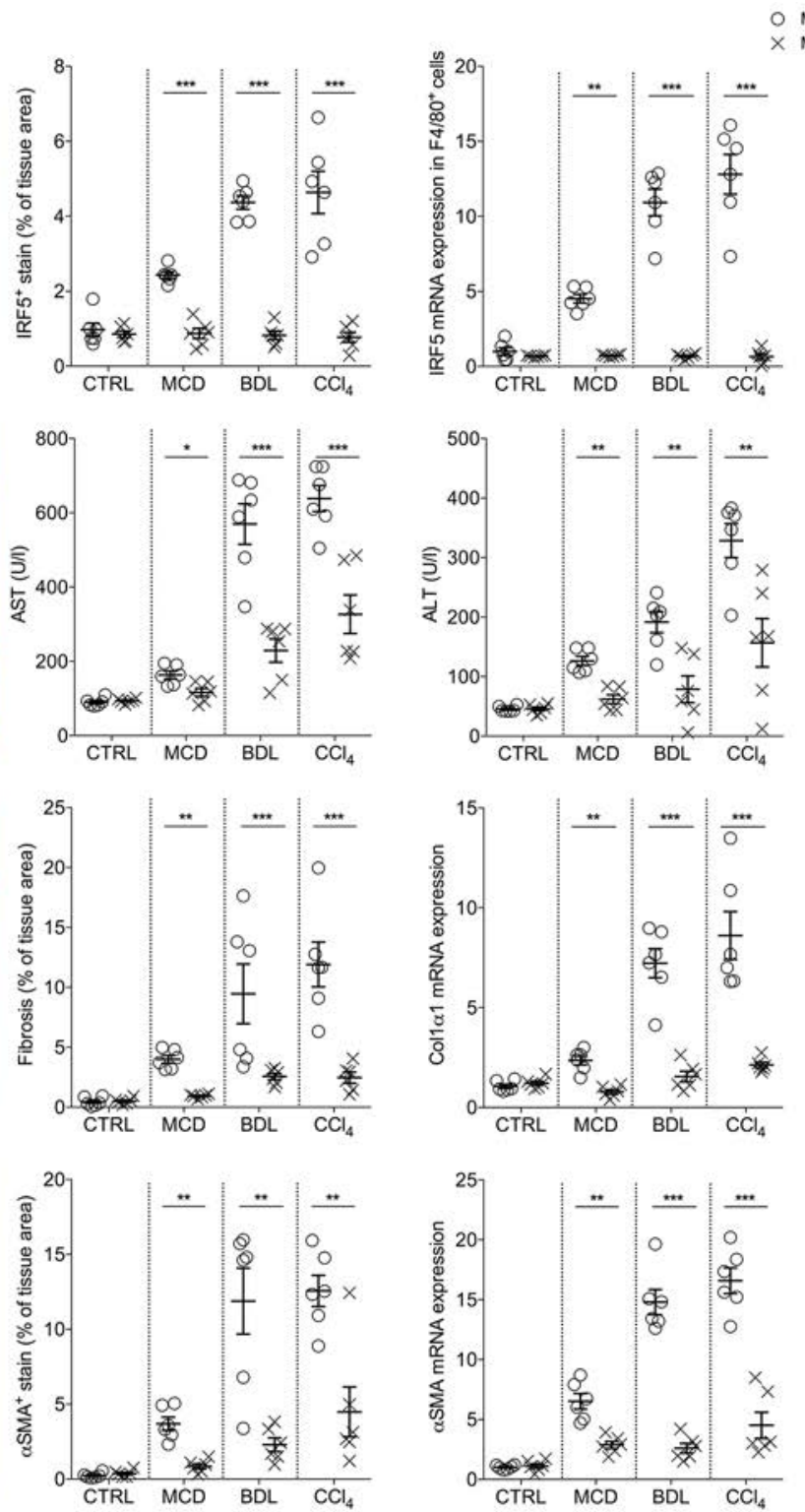

Figure 3. Myeloid deficiency of IRF5 protects mice from liver fibrosis. Wild-type (MWT) mice and mice with myeloid-specific deletion of interferon regulatory factor 5 (IRF5) (MKO) were maintained on a methionine and choline-deficient (MCD) diet or underwent bile duct ligation (BDL) for induction of nonalcoholic steatohepatitis (NASH) or were treated with carbon tetrachloride $\left(\mathrm{CCl}_{4}\right)$ for induction of experimental fibrosis, and compared with mice with healthy liver maintained on a normal chow diet (CTRL). (A) Representative IHC of IRF5 in liver sections from control mice maintained on a normal chow

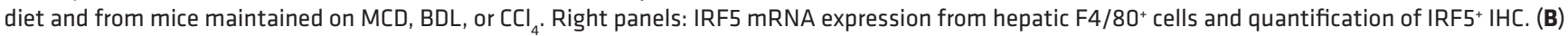
Representative images of H\&E staining of liver sections from CTRL mice maintained on a normal chow diet and from mice maintained on MCD, BDL, or $\mathrm{CCl}_{4}$. Right panels: plasma aspartate transferase (AST) and alanine transferase (ALT) levels of CTRL mice maintained on a normal chow diet and of mice maintained on $\mathrm{MCD}, \mathrm{BDL}$, or $\mathrm{CCl}_{4}$. (C) Representative images of red picrosirius (RP) staining on liver sections from CTRL mice maintained on a normal chow diet and from mice maintained on MCD, BDL, or $\mathrm{CCl}_{4}$. Right panels: quantification of RP staining and liver Col1 $\alpha 1$ mRNA expression. (D) Representative images of $\alpha$ SMA IHC of liver sections from CTRL mice maintained on a normal chow diet and from mice maintained on MCD, BDL, or CCI. Right panels: quantification of $\alpha$ SMA staining and liver $\alpha$ SMA mRNA expression. Scale bars: $100 \mu \mathrm{m} . n=6$ per group. Differences between genotypes determined by unpaired 2 -tailed $t$ test. All values reported as mean \pm SEM. ${ }^{*} P<0.05,{ }^{* *} P<0.01,{ }^{* * *} P<0.001$.

sion of these inflammatory genes was then confirmed by qRT-PCR analysis (Figure 5B). We also observed a significant reduction in CCL2 mRNA expression levels in MKO macrophages (Supplemental Figure 5). In accordance with enriched GO terms for lymphocyte activation, we observed decreased expression of the fibrokines IL13 and IL4 among liver CD4 ${ }^{+} \mathrm{T}$ cells (Figure 5C). Enriched terms among upregulated transcripts represented normal metabolic functions of liver $\mathrm{F} 4 / 80^{+}$cells from MKO relative to MWT mice (Supplemental Table 1 and 2). 
A

A $\quad$ CTRL

MCD

$\mathrm{CCl}_{4}$
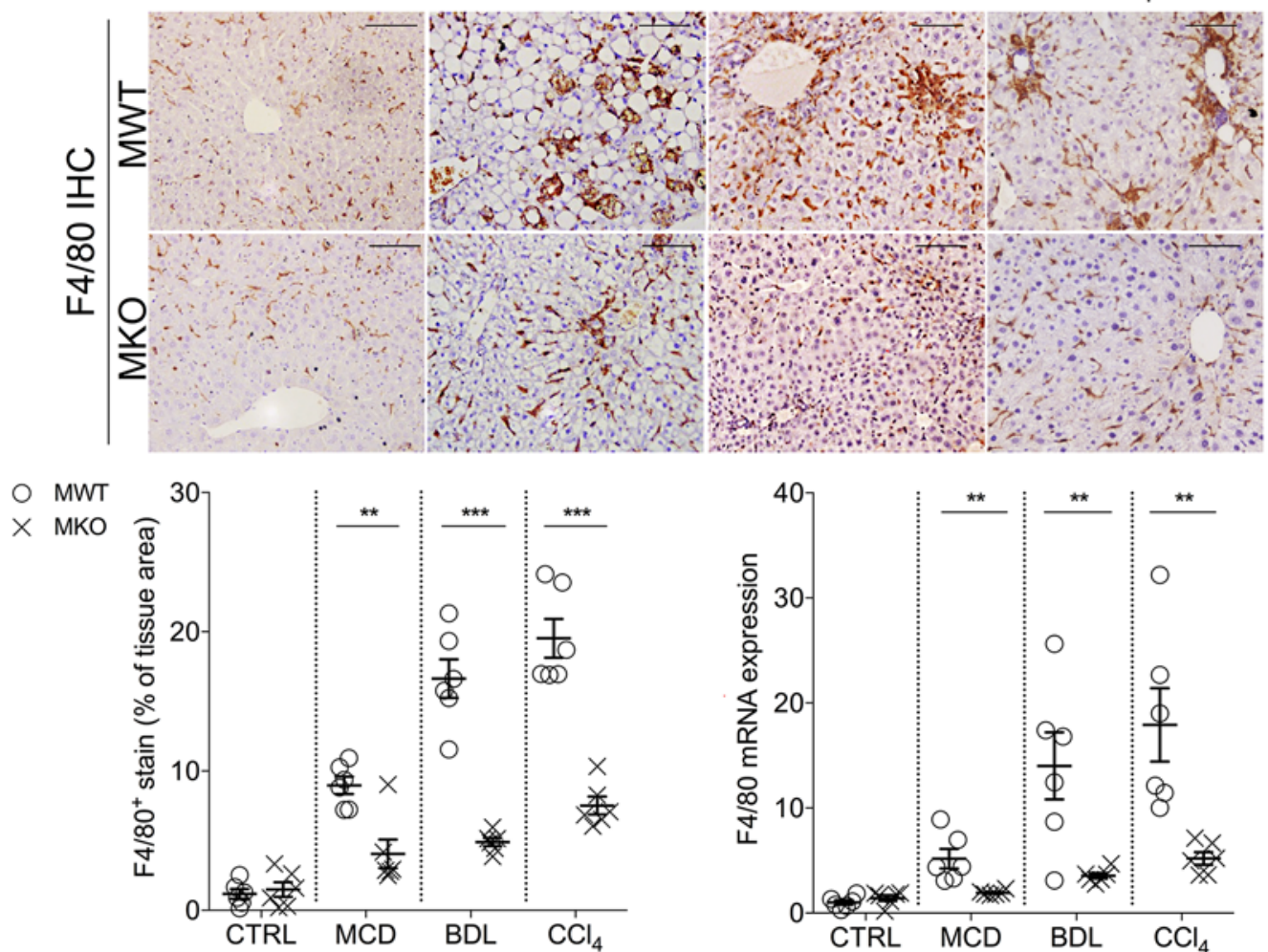

B

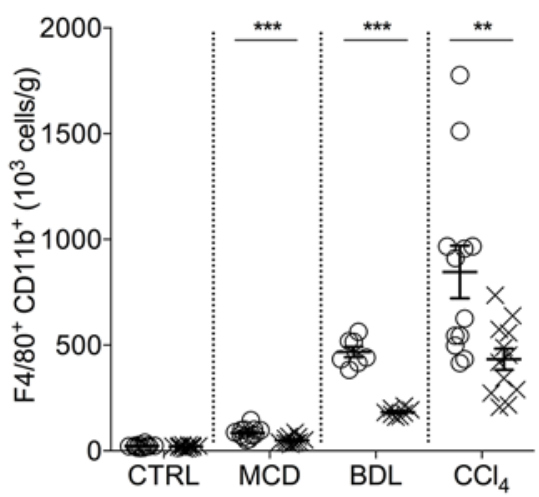

C

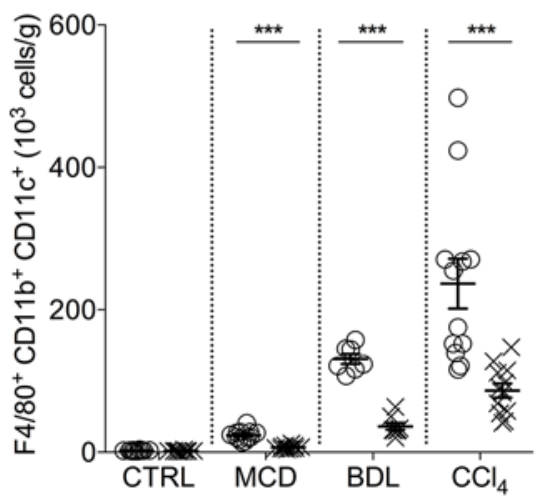

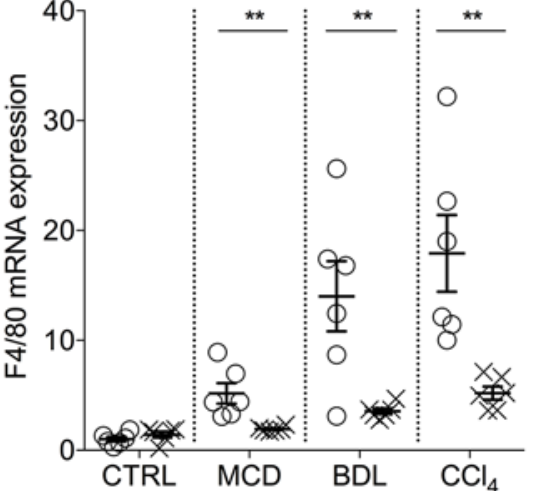

$C D 45^{+}$
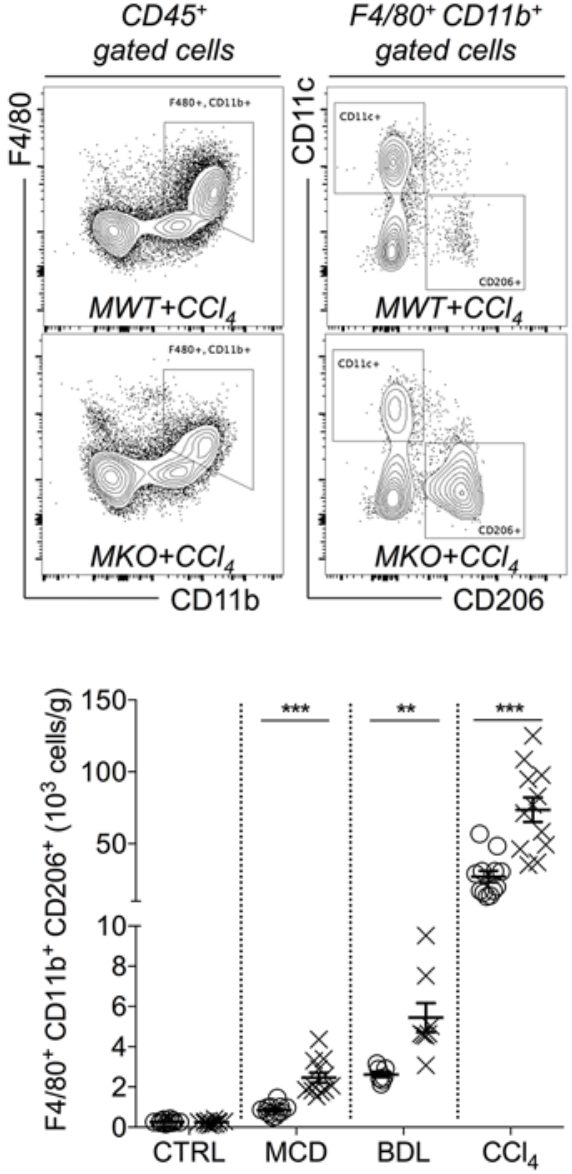

Figure 4. Myeloid deficiency of IRF5 alters macrophage number and phenotype in liver fibrosis. Wild-type (MWT) mice and mice with myeloid-specific deletion of interferon regulatory factor 5 (IRF5) (MKO) were maintained on a methionine and choline-deficient (MCD) diet or underwent bile duct ligation (BDL) for induction of nonalcoholic steatohepatitis (NASH) or were treated with carbon tetrachloride $\left(\mathrm{CCl}_{4}\right)$ for induction of experimental fibrosis and compared with mice with healthy liver maintained on a normal chow diet (CTRL).

(A) Representative images of $F 4 / 80 \mathrm{IHC}$ of liver sections from CTRL mice maintained on a normal chow diet and from mice maintained on $\mathrm{MCD}, \mathrm{BDL}$, or $\mathrm{CCl}_{4}$. Quantification of $\mathrm{F} 4 / 80^{+} \mathrm{IHC}$ and liver F4/80 mRNA expression. (B) Flow cytometric quantification of $\mathrm{F} 4 / 80^{+} \mathrm{CD} 11 \mathrm{~b}^{+}$macrophages among liver mononuclear cells (LMNCs) of CTRL mice maintained on a normal chow diet and of mice maintained on MCD, BDL, or $\mathrm{CCl}_{4}$. (C) Flow cytometric quantification of $\mathrm{CD}_{11 \mathrm{C}^{+}}$or CD206 $6^{+}$macrophages from LMNCS of CTRL mice maintained on a normal chow diet and of mice maintained on MCD, BDL, or $\mathrm{CCl}_{4} \cdot n=$ 6-12 per group. Differences between genotypes determined by unpaired two-tailed $t$ test. All values reported as mean \pm SEM. ${ }^{* *} P<0.01,{ }^{* * *} P$ $<0.001$. 
Myeloid deficiency of IRF5 prevents Fas-linked hepatocyte apoptosis. Apoptosis terms were significantly enriched among downregulated transcripts in the hepatic F4/80+ cells from MKO mice. Thus, we quantified apoptotic and necrotic bodies by TUNEL assay in liver from MWT and MKO mice upon $\mathrm{CCl}_{4}$ treatment. TUNEL ${ }^{+}$staining, histologically localized to hepatocytes, was observed in liver from MWT but not MKO mice, confirming downregulation of cell death (Figure 5D). Among apoptotic pathways, Fas-linked apoptosis had been highlighted through pathway analyses to be downregulated in hepatic macrophages from MKO mice (Figure 5A). We confirmed, by a combination of IHC and qRT-PCR, that FasL expression was reduced in liver sections and in liver macrophages from MKO compared with MWT mice (Figure 5E). We also revealed, through flow cytometry, that hepatic $\mathrm{F} 4 / 80^{+} \mathrm{CD} 11 \mathrm{~b}^{+}$macrophages from IRF5 MKO mice have defective FasL and TNF release compared with MWT mice (Figure 5F). Interestingly, IHC analysis had revealed similar localization of FasL- and TUNEL-positive staining, confirming hepatocytes as targets of Fas-induced apoptosis in MWT mice. These observations were corroborated by decreased expression of Fas (FasL receptor) and increased expression of the antiapoptotic genes BCL2 and BCL- $\mathrm{X}_{\mathrm{L}}$ in hepatocytes from MKO mice compared with MWT littermate controls (Figure 5G).

Apoptosis is an early event that initiates scar tissue deposition and fibrogenesis (21). Thus, we characterized the acute, 24-hour, response to $\mathrm{CCl}_{4}$ administration in MWT and MKO mice. Following acute $\mathrm{CCl}_{4}$ toxicity, we observed disruption of liver structure in MWT mice, the appearance of necrotic/apoptotic regions, and massive hepatocyte ballooning as visualized by H\&E staining. This structural damage was minimal in liver from MKO mice, as corroborated by decreased plasma transaminase levels, which were strongly induced in MWT mice (Figure 6A). Importantly, IRF5 expression was also strongly induced in MWT after acute $\mathrm{CCl}_{4}$ toxicity as quantified by IHC and by qRT-PCR analysis of hepatic F4/80+ cells (Figure 6B). Along with IRF5 induction in MWT mice, we noticed an increased accumulation of $\mathrm{F} 4 / 80^{+} \mathrm{CD} 11 \mathrm{~b}^{+}$macrophages in MWT but not MKO liver (Figure 6C). Additionally, in MKO mice accumulation of inflammatory $\mathrm{CD} 11 \mathrm{c}^{+}$macrophages was reduced, while $\mathrm{CD} 206^{+}$macrophage accumulation was significantly increased. Consistent with this finding, liver macrophages from MKO mice were marked by increased expression of antiinflammatory and immunosuppressive genes, namely TGF $\beta$, IL10, and CD206 (Figure 6D). We also found a strong induction of TUNEL ${ }^{+}$apoptotic/necrotic bodies in MWT but not MKO liver. Apoptosis was confirmed to be Fas induced, with increased FasL ${ }^{+}$staining in MWT but not MKO liver (Figure 6E). Indeed, FasL and TNF release by hepatic F4/80 ${ }^{+} \mathrm{CD} 11 \mathrm{~b}^{+}$macrophages was decreased in MKO liver (Figure 6F), which is concordant with decreased Fas expression in hepatocytes and increased expression of the antiapoptotic genes BCL-X $\mathrm{X}_{\mathrm{L}}$ and BCL2 (Figure 6G). Furthermore, analysis of IRF5 recruitment to DNA in bone marrow-derived macrophages (BMDMs) revealed that IRF5 binds to the upstream region of the FasL gene after stimulation with LPS (Supplemental Figure 6A). FasL mRNA expression was induced in these cells but not IRF5 MKO cells (Supplemental Figure 6B).

Modulation of the adaptive immune responses has previously been attributed to IRF5 activation in macrophages $(16,22)$. Furthermore, in our transcriptome analysis lymphocyte activation was highlighted among enriched terms by differentially expressed transcripts between IRF5 MKO and MWT mice. Indeed, following acute $\mathrm{CCl}_{4}$ toxicity, expression of type 1 (IFN $\gamma$ ) and type 17 (IL17) cytokines was decreased among $\mathrm{CD}^{+} \mathrm{T}$ cells in MKO relative to MWT mice (Figure $6 \mathrm{H}$ ). Furthermore, among $\mathrm{CD}^{+} \mathrm{T}$ cells, we observed increased IL10 expression in MKO mice, concordant with increased accumulation of FoxP3 $3^{+}$ regulatory $\mathrm{CD}^{+} \mathrm{T}$ cells in IRF5 $\mathrm{MKO}$ relative to MWT mice (Figure $6 \mathrm{H}$ ). Rapid polarization of the T cell response toward a type 2 or immunoregulatory response may also contribute to the hepatoprotective phenotype of IRF5 MKO mice.

Collectively, our findings demonstrated that defective release of death effectors FasL and TNF by IRF5-deficient macrophages and their immunosuppressive activation of regulatory $\mathrm{T}$ cells represent key mechanisms of decreased hepatocyte apoptosis.

Myeloid deficiency of IRF5 desensitizes hepatocytes to Fas-induced apoptosis. To confirm the defective induction of Fas-linked apoptosis in MKO mice, we applied a model to directly stimulate the Fas death receptor by administration of an anti-Fas antibody (Jo2 clone). Four hours after administration of Jo2, massive hemorrhaging developed in the liver of MWT mice, as well as elevated plasma transaminase levels, as expected with hepatocyte death. IRF5 MKO mice showed complete protection from Jo2 treatment, and liver structure was maintained, with no change in plasma transaminase levels (Figure 7A). A TUNEL assay confirmed the appearance of apoptotic bodies only in MWT mice and not in MKO mice (Figure 7B). Liver damage seen in MWT mice was associated with an induction of IRF5 expression in F4/80 cells in 
A
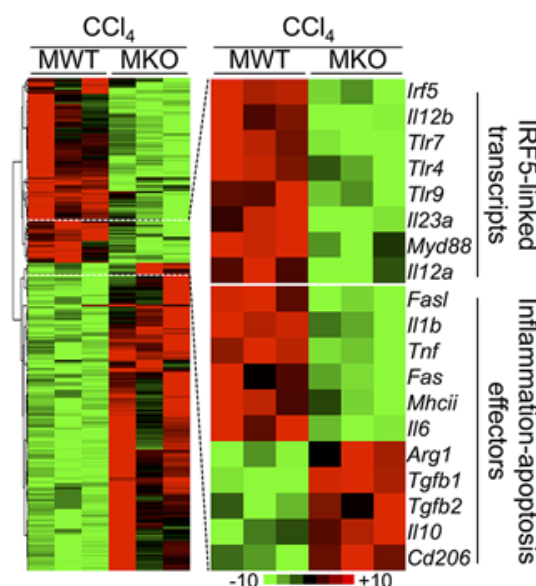

B
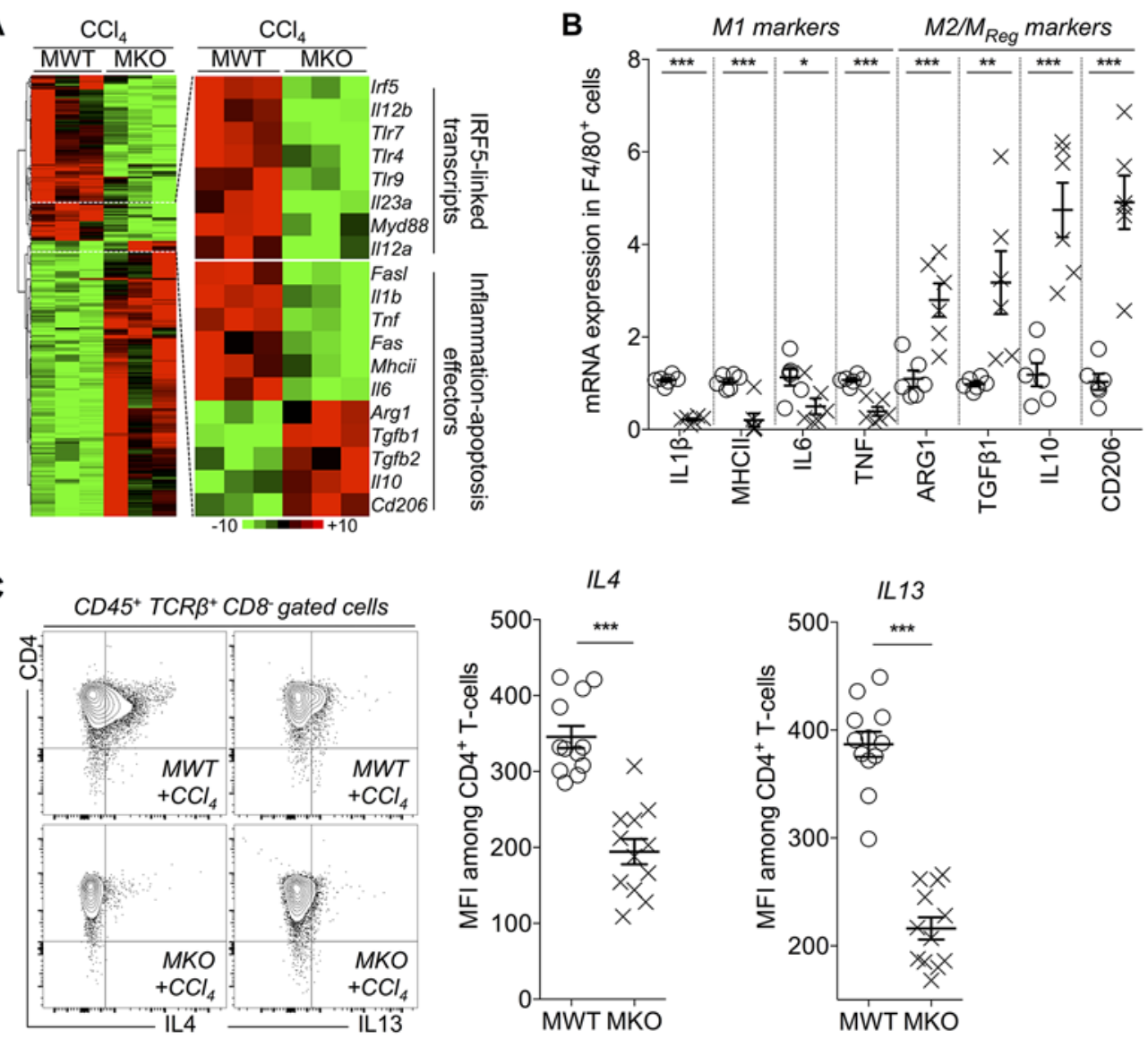

IL4

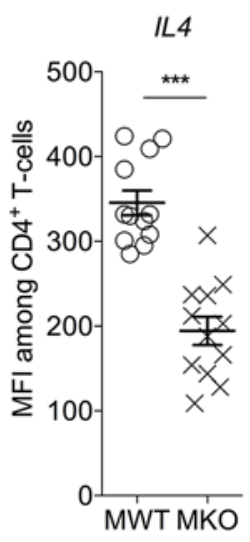

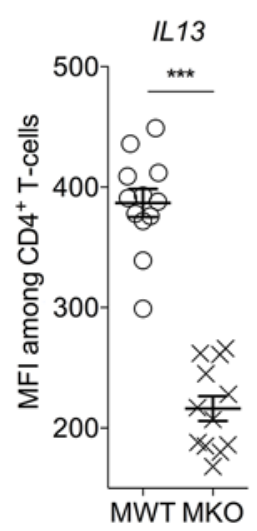

O MWT

$\times$ MKO
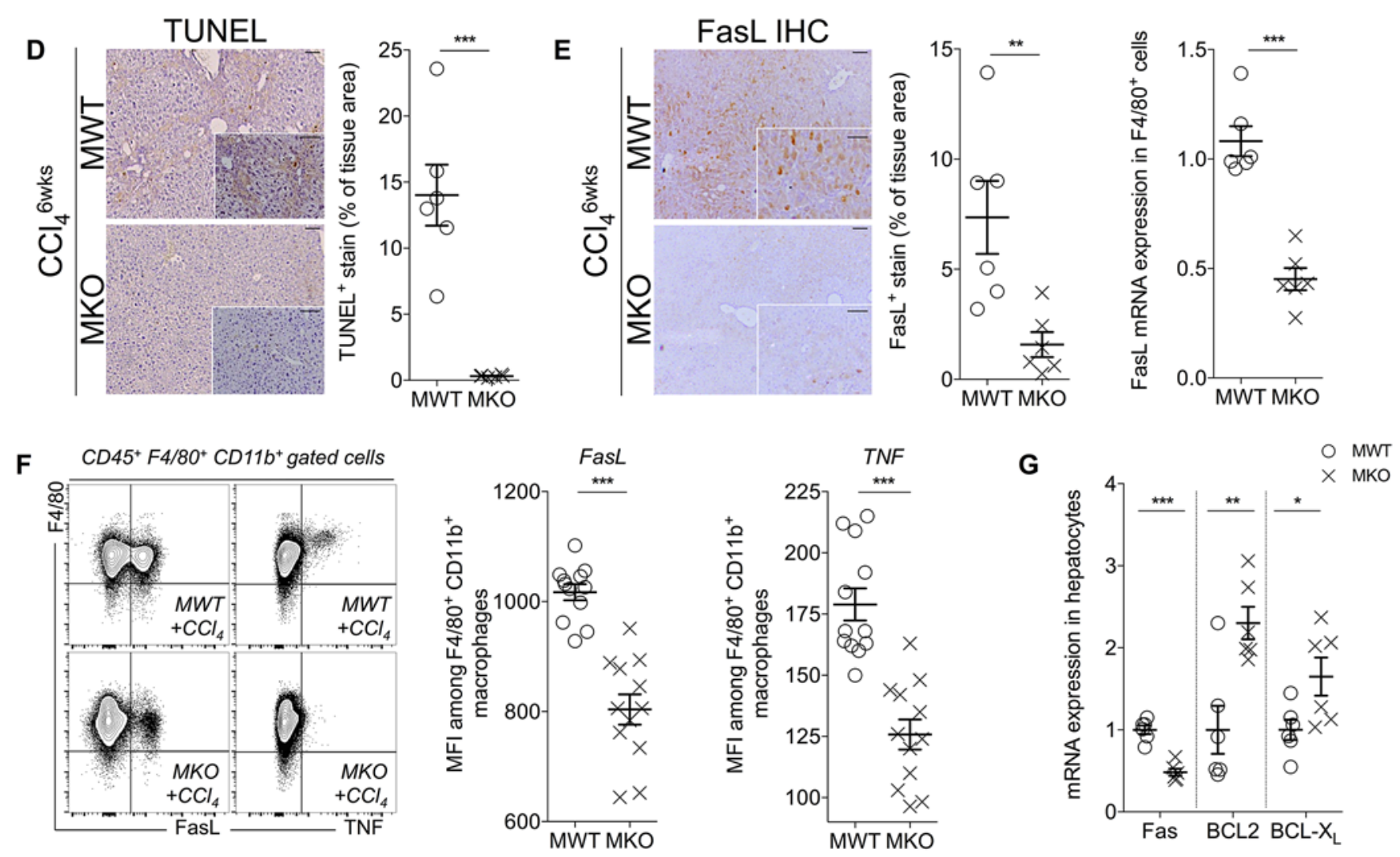
Figure 5. IRF5 knockout alters hepatic macrophage transcriptome response to experimental fibrosis. Wild-type (MWT) mice and mice with myeloid-specific deletion of interferon regulatory factor 5 (IRF5) (MKO) were maintained on carbon tetrachloride (CCI) for induction of experimental fibrosis. (A) Global heatmap and heatmaps of target transcripts representing transcriptomic analysis of F4/80+ liver mononuclear cells (LMNCs) from MWT and MKO mice following $\mathrm{CCl}_{4}$ treatment. (B) qRT-PCR confirmation of target transcript expression from F4/80+ cells of MWT and MKO mice following $\mathrm{CCl}_{4}$ treatment. Markers quantified: IL1 $\beta$, MHC II, IL6, TNF, arginase 1 (ARG1), TGF $\beta 1$, IL10, CD206. (C) Quantification of IL4 and IL13 expression among CD4 ${ }^{+} \mathrm{T}$ cells from LMNCs of MWT and MKO mice following $\mathrm{CCl}_{4}$ treatment. (D) Representative images and quantification of TUNEL ${ }^{+}$apoptotic bodies in liver sections from MWT and MKO mice following $\mathrm{CCl}_{4}$ treatment. (E) Representative IHC of Fas ligand (FasL) in liver sections from MWT and MKO mice following $\mathrm{CCl}_{4}$ treatment. Quantification of $\mathrm{FasL}^{+}$staining of and FasL mRNA expression in hepatic F4/80+ cells. (F) Quantification of FasL and TNF expression among macrophages from LMNCs of MWT and MKO mice following CCl treatment. (G) Quantification of Fas, B cell lymphoma 2 (BCL2), and $\mathrm{BCL}$-extra large $\left(\mathrm{BCL}-\mathrm{X}_{\mathrm{L}}\right)$ mRNA expression in primary hepatocytes from MWT and $\mathrm{MKO}$ mice following $\mathrm{CCl}_{4}$ treatment. For transcriptome analysis, $n$ $=3$ per group; other analyses, $n=6-12$ per group. Scale bars: $100 \mu \mathrm{m}$. Differences between genotypes determined by unpaired 2 -tailed $t$ test. All values reported as mean \pm SEM. ${ }^{*} P<0.05$, ${ }^{* *} P<0.01,{ }^{* *} P<0.001$. Transcriptome filtering based on a detection $P$ value less than 0.05 , difference to control (MWT) of more than 2 -fold change and adjusted $P$ value less than 0.05 .

response to Jo2 treatment, which remained blunted in IRF5 MKO mice (Figure 7C). We then measured the expression of target pro- and antiinflammatory genes expressed from $\mathrm{F} 4 / 80^{+}$cells and found decreased expression of IL1 $\beta$, MHC II, IL6, and TNF and increased expression of TGF $\beta$, IL10, and CD206 (Figure 7D). F4/80 staining and FACS analysis revealed decreased macrophage accumulation (Figure 7E). Furthermore, similar to the responses to $\mathrm{CCl}_{4}$ treatment and confirming the gene expression profile of $\mathrm{F} 4 / 80^{+}$ cells, we observed a predominance of $\mathrm{CD}_{206}{ }^{+}$macrophages in $\mathrm{MKO}$ mice, whereas inflammatory CD $11 \mathrm{c}^{+}$ macrophages predominated among hepatic macrophages of MWT mice (Figure 7F).

In addition to alternative macrophage polarization, the $\mathrm{CD} 4^{+} \mathrm{T}$ cell response was altered in the liver of MKO compared with MWT mice. As observed following acute $\mathrm{CCl}_{4}$ toxicity, the lack of IRF5 in liver macrophages increases differentiation of FoxP3 $3^{+} \mathrm{CD} 4^{+} \mathrm{T}$ cells and expression of IL10 rather than IL17 and IFN $\gamma$ (Figure 7G).

These results demonstrate that IRF5 expression is integral to the induction of apoptosis. In MKO mice we confirmed hepatocyte desensitization to Fas receptor-mediated apoptosis that underpinned by immunosuppressive polarization of IRF5-deficient macrophages and accumulation of regulatory T cells.

Antiapoptotic response in IRF5 knockout is maintained under metabolic stress. To confirm the above findings under metabolic stress, we investigated the apoptotic phenotype and inflammatory response in liver of MWT and MKO mice upon MCD- and BDL- induced fibrosis. Indeed, following BDL and MCD treatment, decreased induction of $\mathrm{TUNEL}^{+}$apoptosis/necrosis was seen in liver sections of MKO compared with MWT mice (Figure 8A). In agreement with previous observations, IHC staining and mRNA expression analysis confirmed FasL involvement in the apoptotic liver phenotype under MCD and BDL conditions. Similar to TUNEL ${ }^{+}$staining, FasL expression was found to be decreased in MKO compared with MWT mice (Figure 8B). Underlying this protective phenotype in $\mathrm{MKO}$ mice, in $\mathrm{F} 4 / 80^{+}$cells expression of immunosuppressive and antiinflammatory genes (IL10, TGF $\beta$ ) was increased, whereas expression of proinflammatory and proapoptotic genes (TNF, FasL) was decreased (Figure 8C). Furthermore, in MKO mice, differentiation of immunosuppressive FoxP3 ${ }^{+} \mathrm{CD}^{+} \mathrm{T}$ cells was increased (Figure $8 \mathrm{D}$ ), corroborating the $\mathrm{CD}^{+} \mathrm{T}$ cell response following $\mathrm{CCl}_{4}$ and Jo2 administration. Hepatoprotection was then confirmed by qRT-PCR analyses of Fas, BCL2, and BCL-X $\mathrm{L}_{\mathrm{L}}$ indeed, Fas expression was decreased in MKO relative to MWT hepatocytes, and antiapoptotic gene expression was increased (Figure 8E).

Collectively, our results demonstrated in both toxic and metabolic contexts that Fas-linked apoptosis is prevented in IRF5 MKO mice. Immunosuppressive activation of F4/80 cells and induction of an immunoregulatory $\mathrm{T}$ cell response is observed in several models of hepatocellular stress and thus strongly implicated in the protective response.

The IRF5/Fas/FasL axis contributes to human hepatic fibrosis and NASH. To evaluate the relationship between IRF5 and the apoptotic role of Fas and FasL in human pathology, we next investigated mRNA expression in human liver biopsies from control patients with normal liver and from patients with fatty liver, NASH, and lesions related to HCV. When stratifying patients by etiology, the expression of both FasL and Fas was significantly induced in NASH and HCV but not in fatty liver or in control liver (Figure 9A). Similar to the expression of IRF5, the expression of both Fas and FasL increased with increasing fibrosis stage (Figure 9B) and was independent of steatosis (Supplemental Figure 7A). We next investigated the correlation of Fas and FasL with several markers of liver damage and found significant positive correlations with plasma transaminases and bilirubin; in contrast, we observed negative correlations with prothrombin time, which is known to decrease with liver damage (Supplemental Figure 

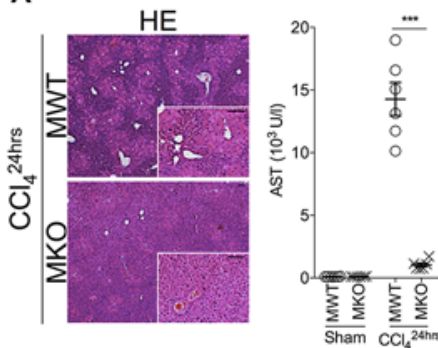

C
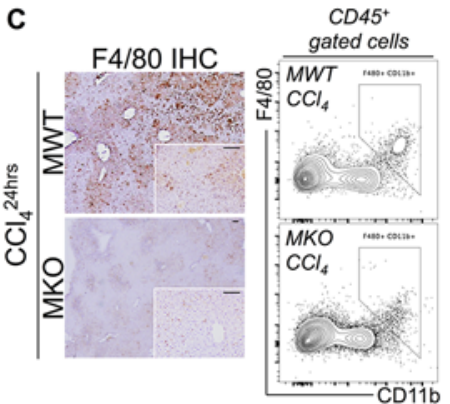
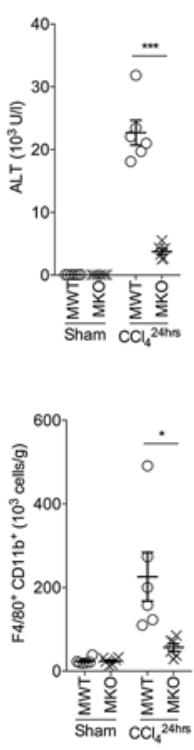

B
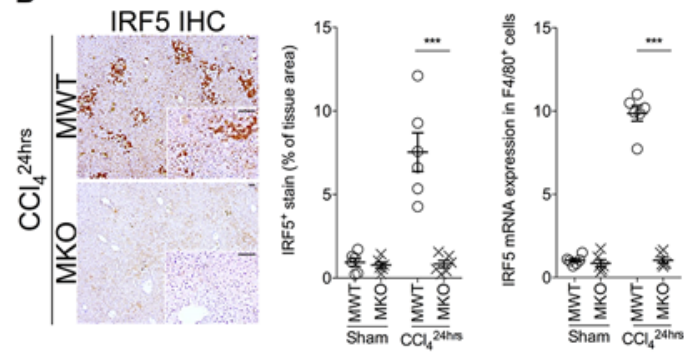

D $\mathrm{F} 4 / 80^{+} \mathrm{CD} 11 \mathrm{~b}^{+}$
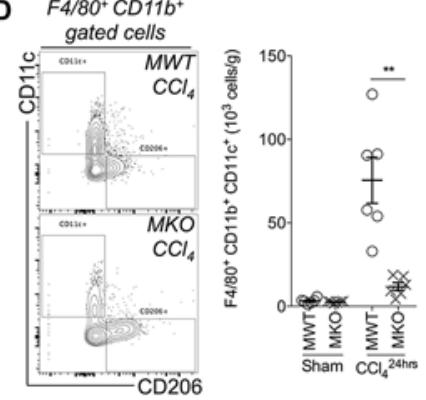
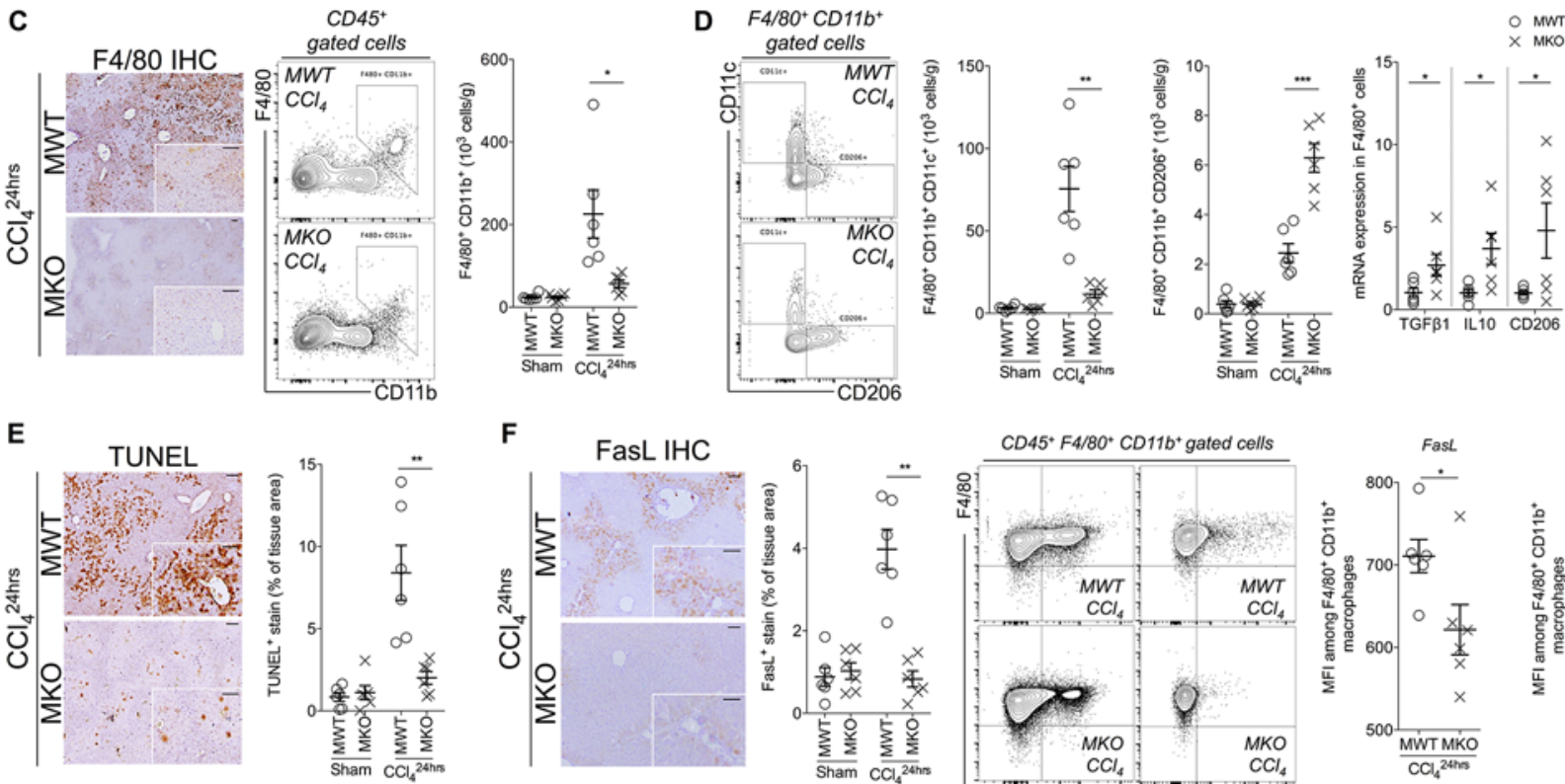

F FasL IHC
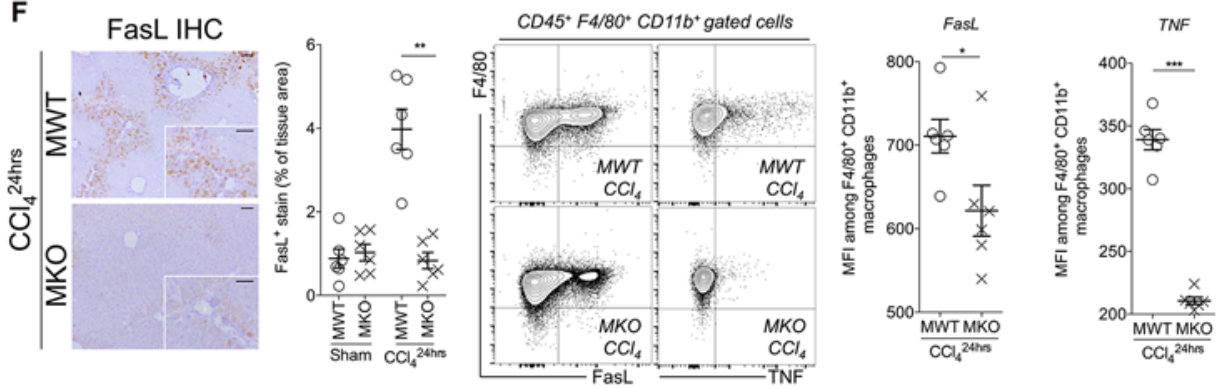

G
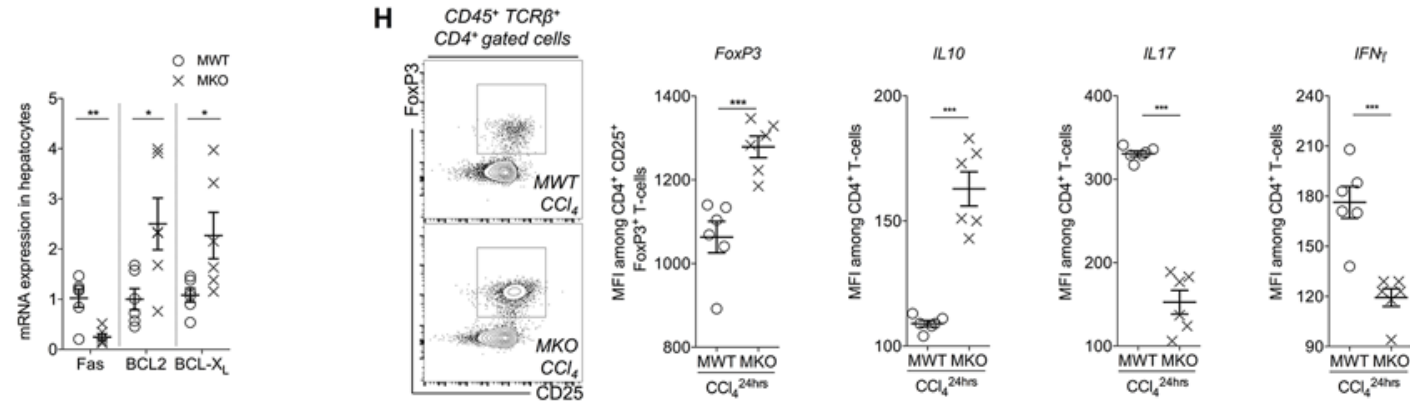

Figure 6. IRF5 knockout prevents apoptosis in acute $\mathrm{CCI}_{4}$ toxicity. Wild-type (MWT) mice and mice with myeloid-specific deletion of interferon regulatory factor 5 (IRF5) (MKO) were administered a single dose of carbon tetrachloride $\left(\mathrm{CCI}_{4}\right)$ for induction of acute toxicity. (A) Representative images of HE staining of liver sections from MWT and MKO mice after acute $\mathrm{CCl}_{4}$ toxicity. Right panels: plasma levels of aspartate transferase (AST) and alanine transferase (ALT) in MWT and MKO mice. (B) Representative images of IRF5 IHC of liver sections from MWT and MKO mice after acute $\mathrm{CCl}_{4}$ toxicity. Right panels: quantification of IRF5 ${ }^{+}$staining and IRF5 mRNA expression in hepatic F4/80+ liver mononuclear cells (LMNCs). (C) Representative images of F4/80 IHC in liver of MWT and MKO mice after acute $\mathrm{CCl}_{4}$ toxicity. Flow cytometric quantification of $\mathrm{F} 4 / 80^{+} \mathrm{CD} 11 \mathrm{~b}^{+}$macrophages among LMNCs. (D) Flow cytometric quantification of CD11c or $\mathrm{CD}_{206}{ }^{+}$macrophages among LMNCs of MWT and MKO mice after acute $\mathrm{CCl}_{4}$ toxicity. Right panel: mRNA expression of TCF $\beta 1$, IL10, and CD206 in F4/80+ LMNCs. (E) Representative images and quantification TUNEL+ apoptotic bodies in liver sections from MWT and MKO mice after acute CCl toxicity. (F) Representative IHC and quantification of Fas ligand (FasL) in liver sections from MWT and MKO mice after acute $\mathrm{CCl}_{4}$ toxicity. Right panels: quantification of FasL and TNF expression among macrophages from LMNCs. (C) Quantification of Fas, B cell lymphoma 2 (BCL2), and BCL-extra large (BCL-X) mRNA expression in primary hepatocytes from MWT and MKO mice after acute $\mathrm{CCl}_{4}$ toxicity. $(\mathrm{H})$ Quantification of FoxP3 $3^{+}$expression in $\mathrm{CD} 25^{+} \mathrm{CD} 4^{+} \mathrm{T}$ cells and of IL10, IL17, and IFN $\gamma$ expression by CD4 ${ }^{+}$T cells among LMNCs from MWT and MKO mice after acute CCI toxicity. Scale bars: $100 \mu \mathrm{m} ; n=6$ per group. Differences between genotypes determined by unpaired 2 -tailed $t$ test. All values reported as mean $\pm \mathrm{SEM}$. ${ }^{*} P<0.05,{ }^{* *} P<0.01,{ }^{* *} P<0.001$.

7B). We next carried out correlative analysis to determine involvement of IRF5 inflammatory activation with Fas-FasL apoptosis in human NAFLD and HCV. We found strong positive correlations of IRF5 with two inflammatory markers, IL $1 \beta$ and TNF $\alpha$, as well as strong positive correlations with Fas and FasL mRNA expression (Figure 9C). 


\section{Table 1. Significantly enriched KEGG terms among downregulated transcripts in IRF5 MKO mice versus MWT mice following experimental fibrosis by $\mathrm{CCl}_{4}$}

\begin{tabular}{lc}
\hline KEGG term & $\boldsymbol{P}$ value \\
$\begin{array}{l}\text { NK cell-mediated } \\
\text { cytotoxicity }\end{array}$ & $4.7 \times 10^{-9}$ \\
$\begin{array}{l}\text { NOD-like receptor } \\
\text { signaling pathway }\end{array}$ & $6.7 \times 10^{-7}$ \\
$\begin{array}{l}\text { Toll-like receptor signaling } \\
\text { pathway }\end{array}$ & $1.0 \times 10^{-6}$ \\
$\begin{array}{l}\text { Apoptosis } \\
\text { Jak/STAT signaling } \\
\text { pathway }\end{array}$ & $1.3 \times 10^{-5}$ \\
\end{tabular}

KEGG, Kyoto Encyclopedia of Genes and Genomes (http://www.genome.jp/kegg); MKO, myeloid-specific knockout; MWT, wild-type; NOD, nucleotide-binding oligomerization domain.
We have demonstrated an IRF5-dependent regulatory cascade that controls response to hepatocellular stress and development of liver fibrosis (Figure 10). We have demonstrated through the use of murine models of hepatocellular stress arising from metabolic stress, toxic stress, and direct cell death receptor stimulation that an IRF5-driven inflammatory response is central to induction of hepatocyte apoptosis and liver damage.

\section{Discussion}

In the spectrum of NAFLD, progression from benign steatosis to pathological steatohepatitis and fibrosis is largely driven by inflammation. Furthermore, inflammatory activation in NASH is well documented as part of the systemic inflammation that characterizes obesity and type 2 diabetes. Both inflammatory processes have been shown to be ameliorated by IRF5 deficiency $(16,23)$. In this study, we report an IRF5-driven pathway central to steatohepatitis and liver fibrogenesis in mice and humans. Through several models of hepatocellular stress, we demonstrate therapeutic knockdown of macrophage-derived IRF5 that prevents hepatocyte apoptosis by protective induction of the immune system. These events represent early immunosuppressive signaling that interferes with chronic wound healing and liver fibrogenesis.

In the absence of macrophage-derived IRF5 in the liver, antiinflammatory and immunosuppressive macrophage polarization predominates, allowing release of antiinflammatory cytokines (IL10, TGF $\beta$ ) and limiting chemokine production (CCL2) and recruitment of monocytes (24). IRF5-deficient liver macrophages are also characterized by decreased death effector release (FasL, TNF). Conversely, IRF5-competent macrophages undergo proinflammatory and proapoptotic polarization under stress, leading to increased inflammatory cytokines and death effector release. Induction of hepatocyte apoptosis perpetuates scarring fibrogenesis, with functional consequences on the liver. Increased macrophage-derived TGF $\beta$ expression favors $\mathrm{CD} 4^{+} \mathrm{FoxP}^{+} \mathrm{T}$ cell differentiation and IL10 release (Figure 10). Under hepatocyte stress in IRF5 deficiency, rapid IL10 secretion from macrophages and CD4+ $\mathrm{T}$ cells promotes antiapoptotic signaling (BCL2, BCL- $\mathrm{X}_{\mathrm{L}}$ ) in hepatocytes, maintaining their survival and preventing wound healing fibrogenesis, as previously described (25) (Figure 10).

Underpinning decreased fibrosis in IRF5 deficiency is decreased HSC activation. Activation of HSCs is a necessary event in extracellular matrix deposition during fibrogenesis (20). Macrophage inflammatory activation and cytokine release under hepatocellular stress are major mechanisms of HSC activation. The strongest known cytokine inducers of HSC activation are IL1 $\beta$, TNF, and TGF $\beta$ (26). TGF $\beta$ is widely known for its profibrogenic properties; pathological effects of TGF $\beta$ in liver disease have been well documented, with several studies suggesting therapeutic intervention with TGF $\beta$ signaling (27). However, TGF $\beta$ is also a powerful antiinflammatory and immunosuppressive cytokine; thus, attention is increasingly paid to temporal considerations and cell type-specific effects of TGF $\beta$ signaling (25). We have previously demonstrated transcriptional regulation of TGF $\beta$ expression by IRF5 in adipose tissue macrophages (16). The repressive action of IRF5 on TGF $\beta$ expression is reproduced in this study, indicating consistent regulation between tissues, although the effects induced are tissue-specific. These observations emphasize the importance of tissue microenvironments in dictating physiological and pathological cytokine roles. Similar reports have been made regarding IL4 and IL13 - metabolically protective in adipose tissue yet deleterious in the liver (28-30).

Hepatocyte apoptosis is a feature common across a number of liver etiologies. Fibrogenesis is the end product of excessive hepatocellular stress and hepatocyte death that perpetuates inflammation and HSC activation (26). Therapeutic strategies aiming to regulate the balance between anti- and proapoptotic signaling have been previously proposed $(3,4,31-33)$. The apoptotic process is mediated by death receptor
Table 2. Significantly enriched GO terms among downregulated transcripts in MKO mice versus MWT mice following experimental fibrosis by $\mathrm{CCl}_{4}$

\begin{tabular}{lc}
\hline GO term & $P$ value \\
Lymphocyte activation & $7.2 \times 10^{-13}$ \\
Immune effector process & $3.8 \times 10^{-6}$ \\
Apoptosis & $1.4 \times 10^{-5}$ \\
Inflammatory response & $4.2 \times 10^{-3}$ \\
Induction of apoptosis & $1.9 \times 10^{-2}$
\end{tabular}

CO, Gene Ontology (http://geneontology.org); MKO, myeloid-specific knockout; MWT, wild-type. 
A

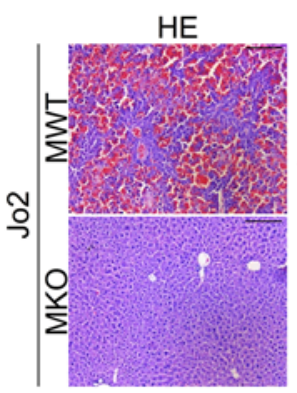

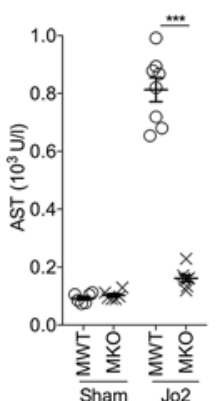

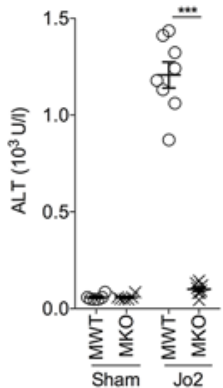

B

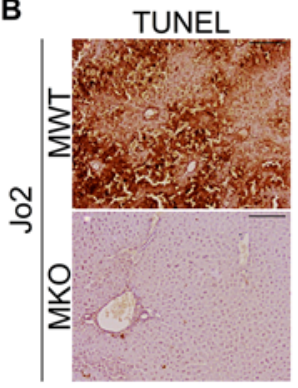

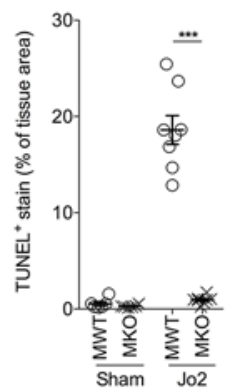

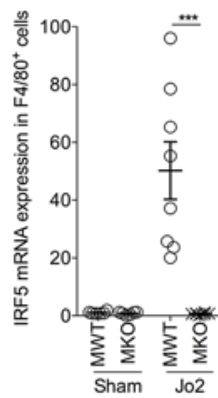

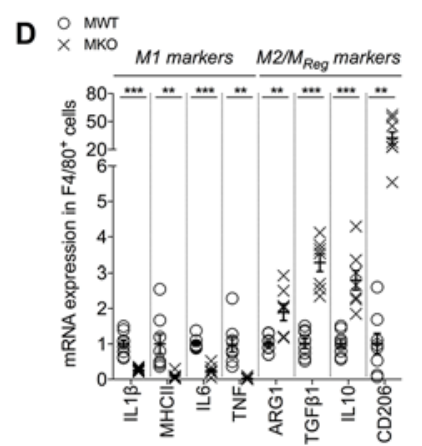

E

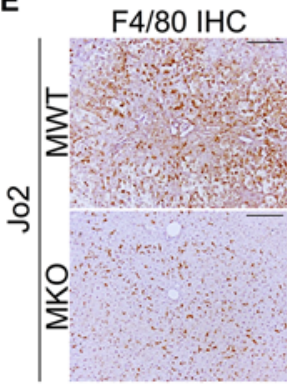

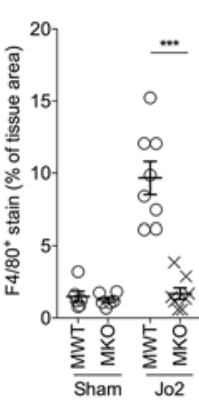

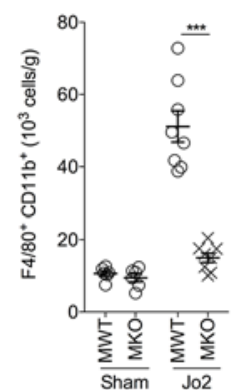

F

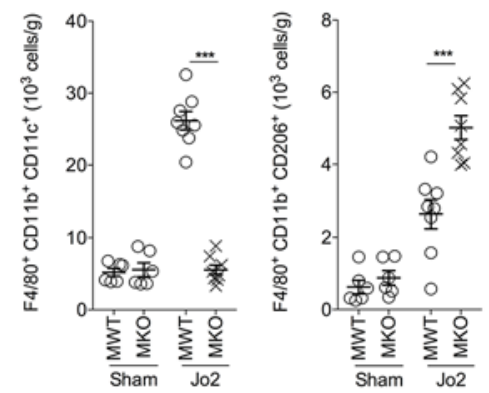

G
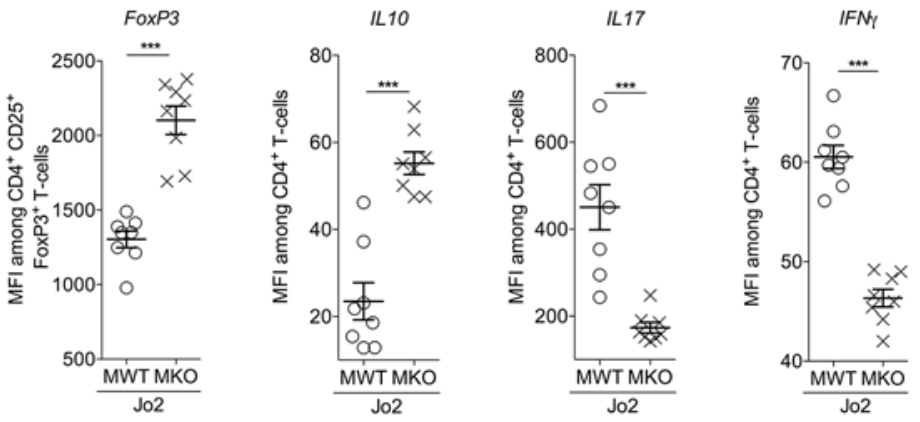

Figure 7. IRF5 deficiency desensitizes hepatocytes to Fas receptor stimulation. Wild-type (MWT) mice and mice with myeloid-specific deletion of interferon regulatory factor 5 (IRF5) (MKO) were administered an anti-Fas antibody (Jo2) for induction of apoptosis. (A) Representative images of H\&E staining of liver sections from MWT and MKO mice after Jo2 administration. Right panel: plasma levels of aspartate transferase (AST) and alanine transferase (ALT) in MWT and MKO mice. (B) Representative images and quantification TUNEL apoptotic bodies in liver sections from MWT and MKO mice after Jo2 administration. (C) Quantification of IRF5 mRNA expression in hepatic F4/80+ liver mononuclear cells (LMNCs) in MWT and MKO mice after Jo2 administration. (D) Quantification of inflammatory marker expression in F4/80+ LMNCs in MWT and MKO mice after J02 administration. Markers quantified: IL1 1 , MHC II, IL6, TNF, arginase 1 (ARG1), TCF $\beta 1$, IL10, CD206. (E) Representative images and quantification of F4/80+ IHC staining of liver sections from Jo2-treated MWT and MKO mice. Flow cytometric quantification of F4/80+ CD11b+ macrophages among LMNCs of Jo2-treated MWT and MKO mice. (F) Flow cytometric quantification of $\mathrm{CD}_{11 c^{+}}$and $\mathrm{CD} 206^{+}$macrophages among LMNCs of Jo2-treated MWT and MKO mice. (G) Quantification of FoxP3 expression in CD25+CD4 ${ }^{+}$T cells, and of IL10, IL17, IFN $\gamma$ expression by CD4 ${ }^{+}$T cells among LMNCs from MWT and MKO mice after Jo2 administration. Sham-treated mice: $n=6$ per group; Jo2-treated mice: $n=6-8$ per group. Scale bars: $100 \mu \mathrm{m}$. Differences between genotypes determined by unpaired 2-tailed $t$ test. All values reported as mean $\pm \mathrm{SEM} .{ }^{* *} P<0.01,{ }^{* * *} P<0.001$.

stimulation and the formation of complexes cleaved at initiator then executioner caspases $(33,34)$. Once the terminal caspase is cleaved, apoptosis is underway. Checkpoints, such as BCL2, can inhibit progression to the terminal caspase or maintain cell structural integrity through other means, thus promoting cell survival (35). Three effector groups are involved in apoptosis: extrinsic factors (viruses, xenobiotics, etc.), immune factors (receptor ligands), and intrinsic factors (bile acids, free fatty acids, etc.) (4). By the application of metabolic stress (MCD, BDL), toxic stress $\left(\mathrm{CCl}_{4}\right)$, and direct death receptor stimulation (Jo2), we have demonstrated an effector-independent mechanism that promotes hepatocyte survival through the myeloid deficiency of IRF5.

IL10 and its targets are instrumental in the protective phenotype observed in IRF5 deficiency, due to the powerful antiapoptotic and antifibrogenic properties of this cytokine. Other studies have demonstrated that 
A
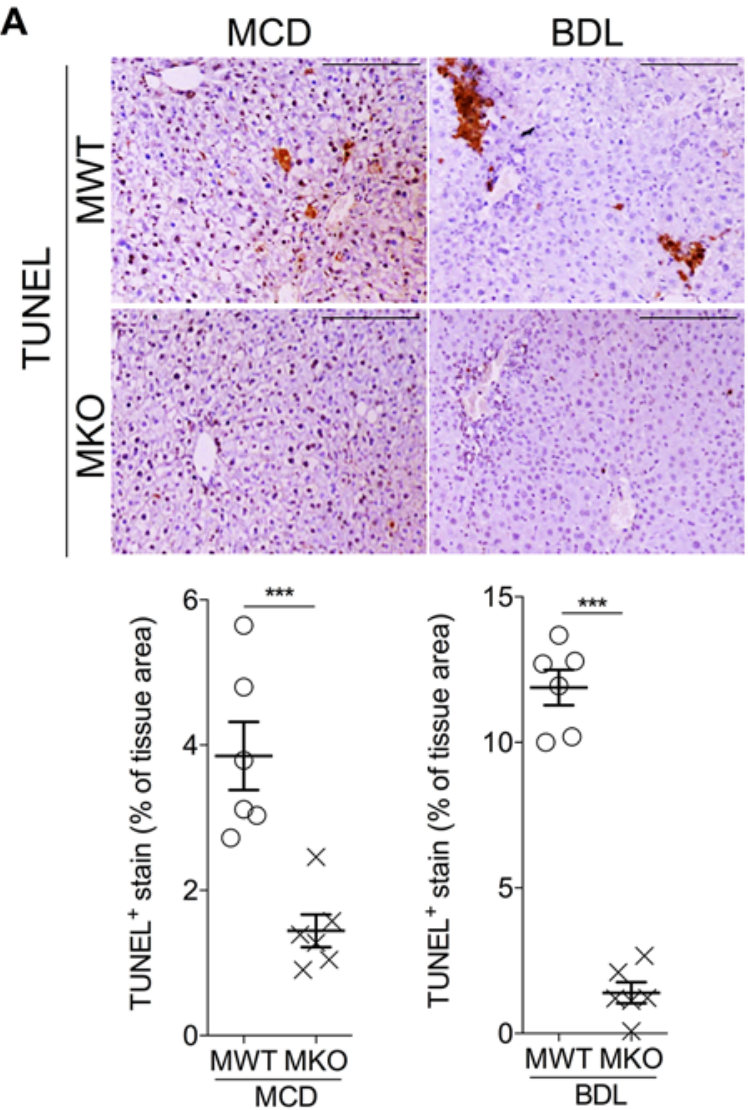

C
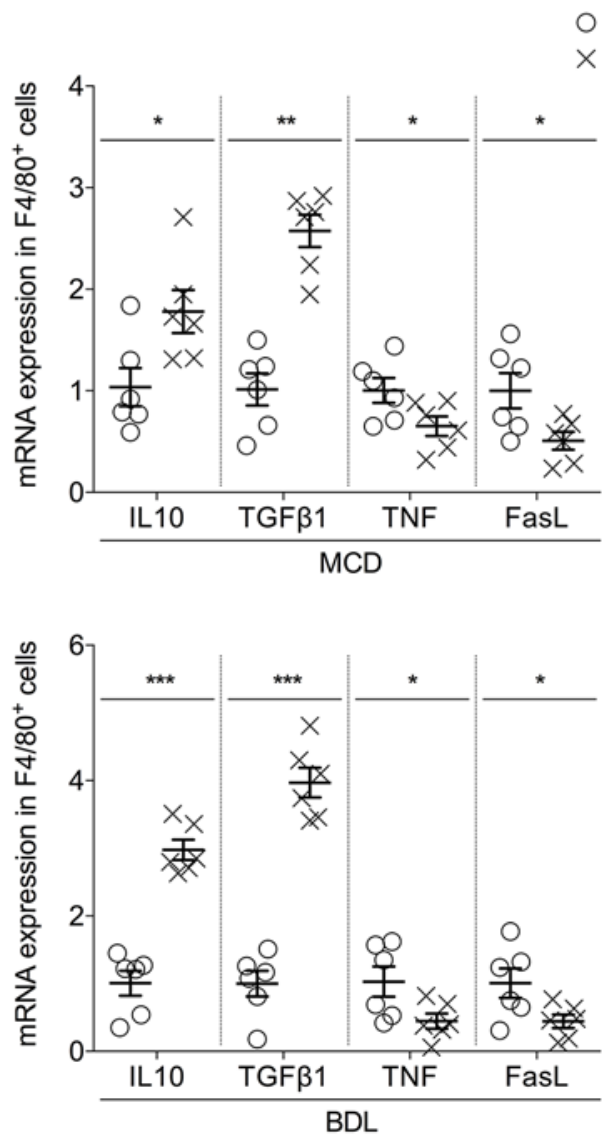
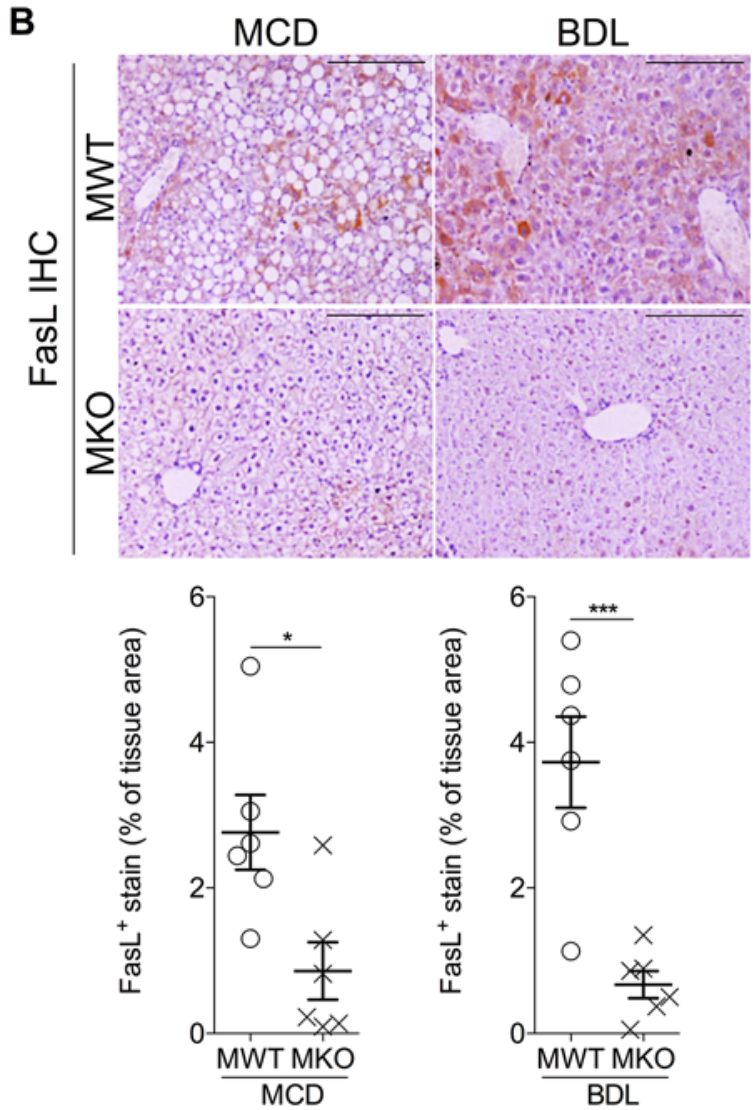

○ MWT
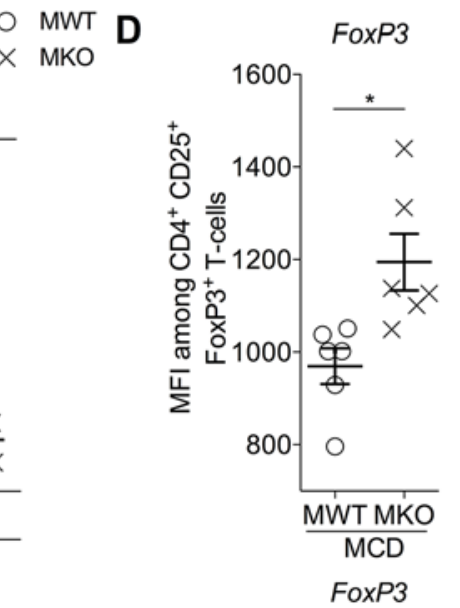

E

O MWT

$\times$ MKO
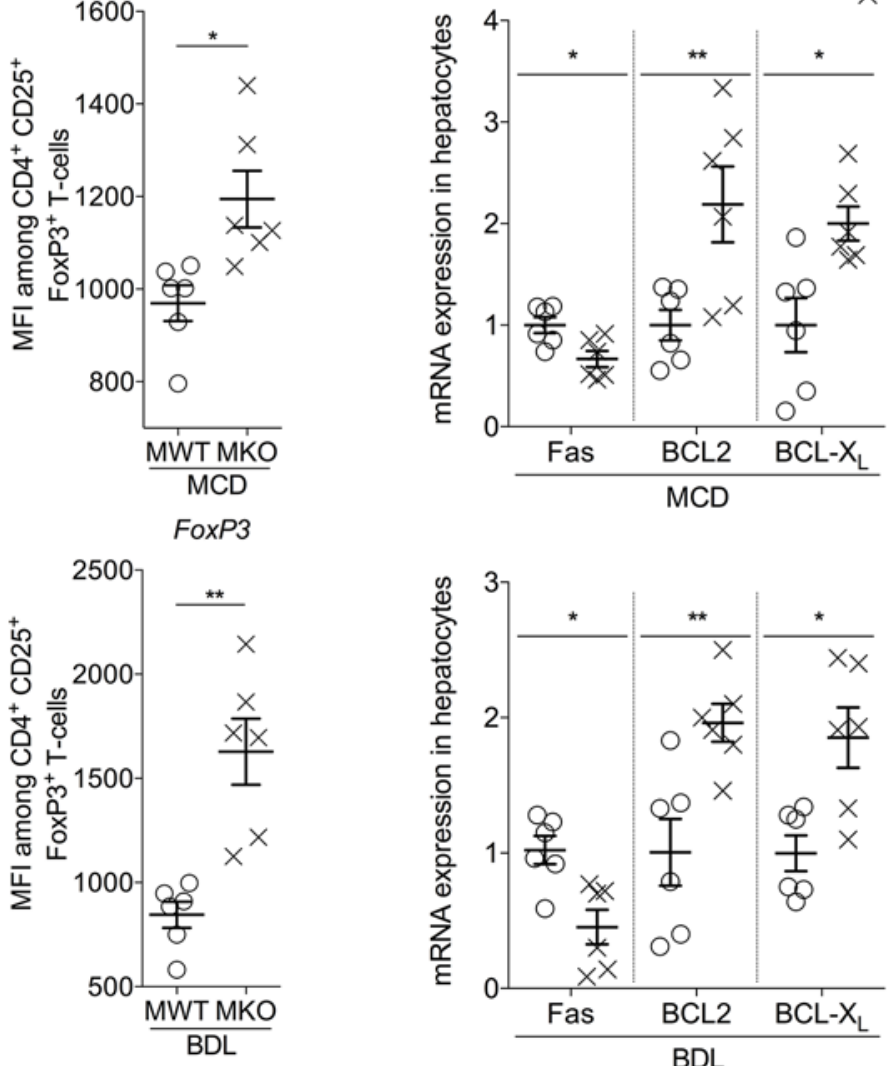
Figure 8. Myeloid deficiency of IRF5 protects from Fas-linked apoptosis in murine NASH. Wild-type (MWT) mice and mice with myeloid-specific deletion of interferon regulatory factor 5 (IRF5) (MKO) were maintained on a methionine and choline-deficient (MCD) diet or underwent bile duct ligation (BDL) for the induction of nonalcoholic steatohepatitis (NASH). (A) Representative images and quantification of IHC TUNEL apoptotic bodies in liver sections from MWT and MKO mice upon MCD and BDL. (B) Representative images and quantification of Fas ligand (FasL) IHC in liver sections from MWT and MKO mice upon MCD and BDL. (C) Top panel: quantification of mRNA expression of IL10, TCF $\beta 1$, TNF, and FasL in F4/80+ liver mononuclear cells (LMNCs) from MWT and MKO mice upon MCD and BDL. Bottom panel: quantification of IL10, IL17, and IFN $\gamma$ expression by CD4+ T cells among LMNCs from MWT and MKO mice upon MCD and BDL. (D) Quantification of FoxP3 $3^{+}$expression in CD25+CD4+ T cells. (E) Quantification of Fas, B cell lymphoma 2 (BCL2), and BCL-extra large $\left(B C L-X_{L}\right)$ mRNA expression in primary hepatocytes from MWT and MKO mice following $\mathrm{CCl}_{4}$ treatment. $n=6$ per group. Scale bars: $100 \mu \mathrm{m}$. Differences between genotypes determined by unpaired 2 -tailed $t$ test. All values reported as mean \pm SEM. ${ }^{*} P<0.05,{ }^{* *} P<0.01,{ }^{* * *} P<0.001$.

mice deficient in IL10 show an aggravated liver phenotype under experimental fibrosis relative to WT mice (36). IL10 expression per se is sufficient to prevent hepatocyte death from caspase-dependent apoptosis by strong induction of BCL2 family members (35). BCL2 and BCL- $\mathrm{X}_{\mathrm{L}}$ are critical factors that promote hepatocyte survival by direct interference with caspase cleavage $(25,34)$. Furthermore, BCL2 family members have been subject to numerous loss- and gain-of-function studies demonstrating their requirement to maintain hepatocyte survival (37). Indeed, under hepatocellular stress in IRF5 MKO, we demonstrate increased hepatocyte expression of BCL2 and BCL- $\mathrm{X}_{\mathrm{L}}$ concordant with increased IL10 expression from leukocytes.

The essential role of $\mathrm{KCs}$ in inducing hepatocyte apoptosis is well documented. Sato et al. (7) demonstrated that chlodronate liposome abolition of $\mathrm{KCs}$ prevents apoptosis following acute $\mathrm{CCl}_{4}$ toxicity; they also demonstrated the therapeutic neutralization of FasL and TNF through antibody administration. We demonstrate that altering macrophage inflammatory polarization is sufficient to ameliorate hepatic response to $\mathrm{CCl}_{4}$ toxicity and in turn prevents experimental fibrosis. In response to Fas stimulation, IRF5-deficient macrophages undergo antiinflammatory polarization, inducing IL10 secretion from $\mathrm{CD} 4^{+} \mathrm{T}$ cells and their rapid immunosuppressive differentiation. Furthermore, upon Fas stimulation, macrophage deficiency in IRF5 renders hepatocytes completely resistant to apoptosis; this resistance has been previously demonstrated in mice with global IRF5 knockout (38). These findings confirm that IRF5 as a key component of the inflammatory process that drives hepatocyte death in mice.

The importance of Fas-induced apoptosis has been previously implicated in human hepatitis, with previous work focusing on Fas induction under advanced hepatopathology, where its expression as an early marker in hepatocarinogenesis is proposed (31). However, few groups have investigated the Fas-FasL system as an early or direct marker of fibrogenesis in NASH. Indeed Fas and FasL expression are induced in NASH and fibrosis in our cohort, similar to IRF5 expression. Most interesting are the strong correlations established between IRF5 expression and the expression of Fas, FasL, and inflammatory markers.

In summary, we have established a key role for IRF5 in human liver fibrosis. Our studies highlight the IRF5-dependent pathway as instrumental in susceptibility to hepatocyte apoptosis (Figure 10). The protective effect of IRF5 inhibition is rooted in antiinflammatory macrophage activation, IL10 and TGF $\beta$ release, and rapid expansion of immunosuppressive $\mathrm{CD} 4^{+} \mathrm{FoxP}^{+} \mathrm{T}$ cells. IL10 release inhibits Fas-dependent apoptosis in hepatocytes, and early hepatoprotection prevents progression of NAFLD beyond benign steatosis, preventing inflammation and fibrogenesis in the liver. Thus, the modulation of IRF5 activity is an increasingly promising approach to experimental therapeutics in fibro-inflammatory liver disease.

\section{Methods}

Human tissue. Liver samples were obtained from 36 patients by surgical resection. Four main groups of patients were analyzed according to their liver lesions: (a) normal liver (control), (b) steatosis without steatohepatitis (fatty liver), (c) steatosis with steatohepatitis (NASH), and (d) necro-inflammatory lesions related to HCV infection (Table 3). Only patients without a clinical history of cirrhosis were included. Control samples were obtained at distance from benign hepatic tumor or from patients with colorectal carcinoma; inclusion as controls was based on histological and serological parameters. NAFLD and HCV samples were obtained at distance from hepatocellular carcinoma. Liver lesions were scored according to the steatosis, activity, and fibrosis (SAF) score (39). Fibrosis stage for NAFLD and HCV was assessed according to the Kleiner and METAVIR systems, respectively (40). All patients signed an informed consent form, and the local ethics committee approved the study. Tissues were frozen or formalin-fixed and paraffin-embedded for RNA extraction and pathological evaluation, respectively.

Murine models of liver injury. Mice were maintained and housed under conventional conditions at the Biological Services Unit of the University of Paris (UPMC). Up to 5 mice were housed per cage under 
A

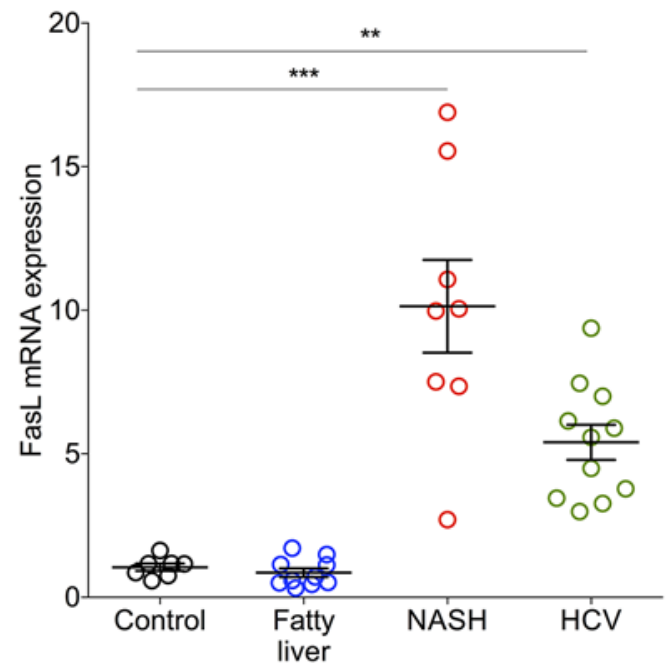

B

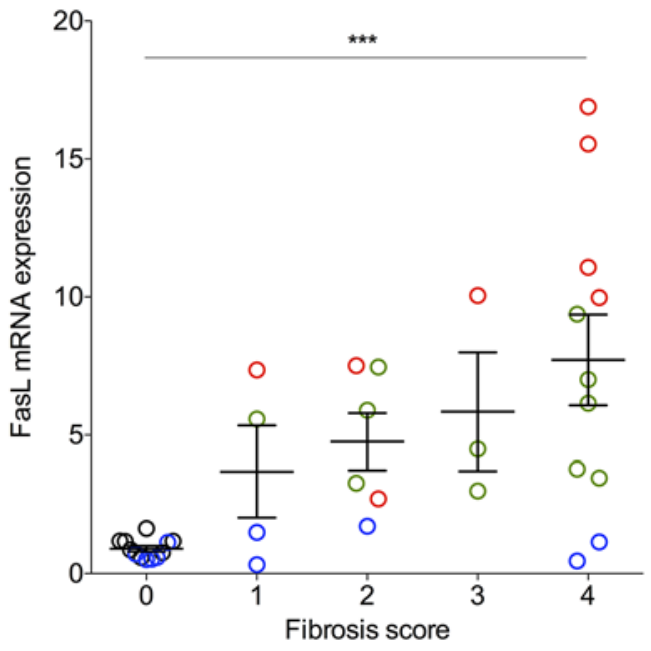

C

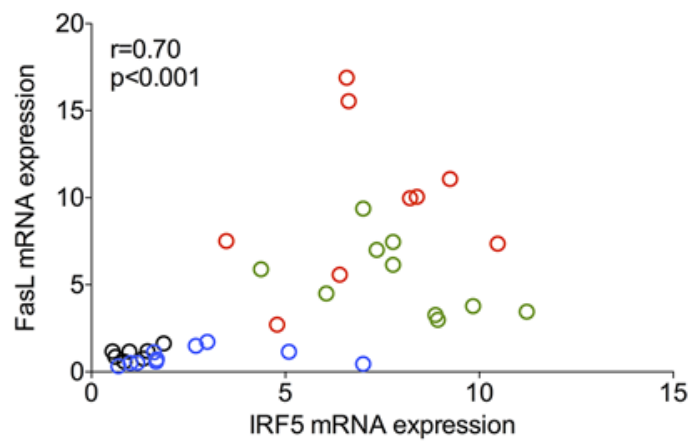

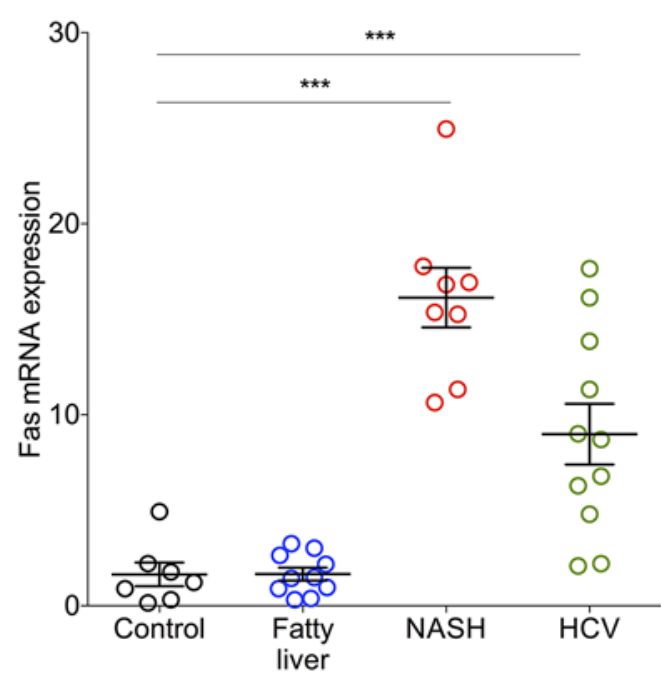

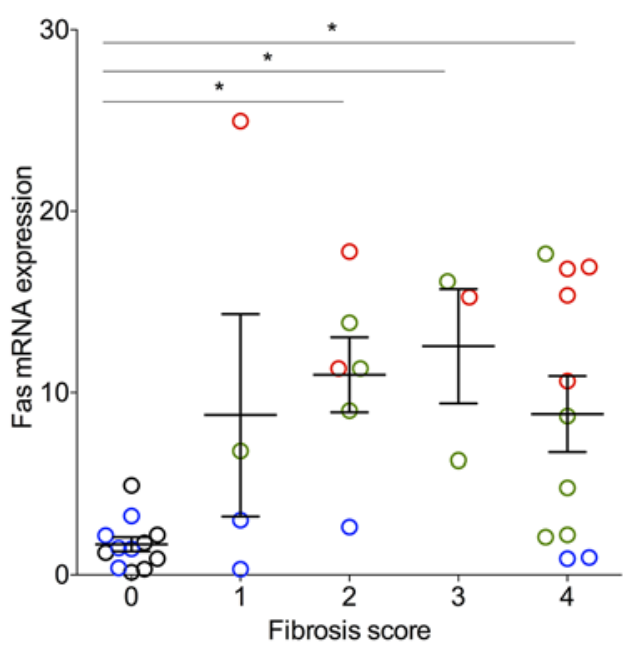

O Control

O Fatty liver

O NASH

O HCV

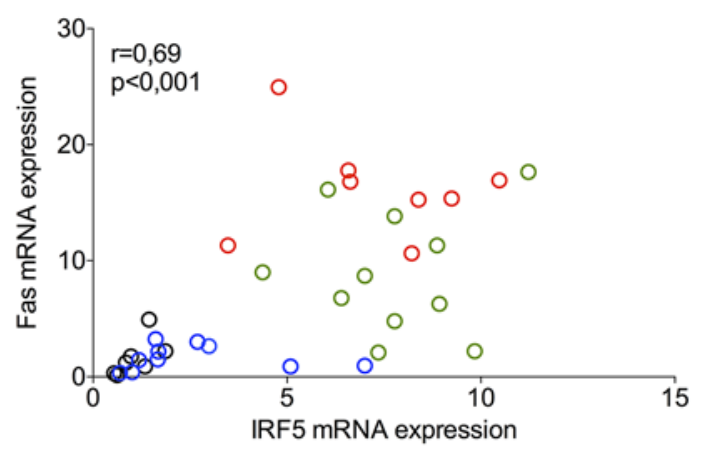

O Control

Fatty liver

O NASH

O $\mathrm{HCV}$

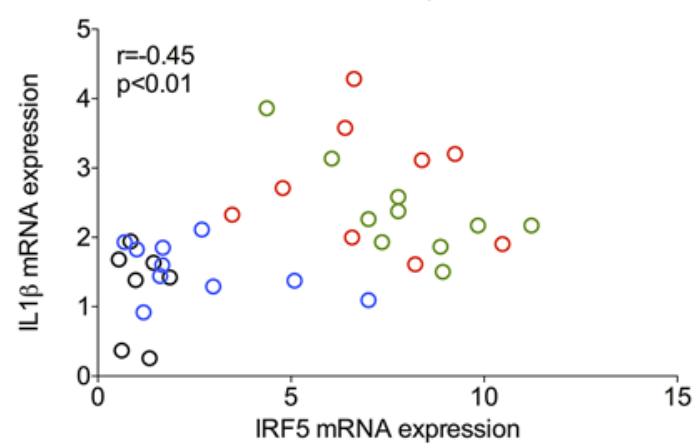


Figure 9. The IRF5/FasL/Fas axis is implicated in pathogenesis of human NASH. (A) Fas and Fas ligand (FasL) mRNA expression in liver biopsies from control patients with normal liver $(n=7)$ and patients with fatty liver $(n=10)$, nonalcoholic steatohepatitis (NASH; $n=8)$, and hepatitis C virus infection (HCV; $n=11$ ). (B) Fas and FasL mRNA expression in the same cohort of patients $(n=36)$ stratified by fibrosis stage. (C) Correlative analyses of interferon regulatory factor 5 (IRF5) mRNA expression with mRNA expression of Fas, FasL, IL1 $\beta$, and TNF in the same cohort of patients ( $n=36$ ). Differences between patient groups determined by 1-way ANOVA. Correlative analyses were assessed by Spearman's test. All values reported as mean \pm SEM. ${ }^{*} P<0.05,{ }^{* *} P<0.01,{ }^{* *} P<0.001$.

alternating 12-hour light/12-hour dark cycles temperature controlled at $23^{\circ} \mathrm{C}$. To generate mice with a myeloid-specific deletion of IRF5, IRF5 ${ }^{f l f l}$ mice (C57BL/6-Irf5 $5^{\text {tm IPpr }} / \mathrm{J}$; stock no. 017311) were crossed with

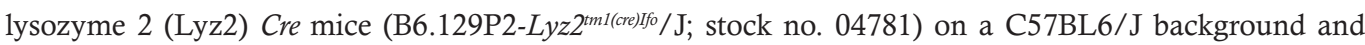
backcrossed for 10 generations before application to studies. Both IRF5 $5^{f / f l}$ mice and Lyz2 Cre mice were obtained from the Jackson Laboratory. Mice carrying mutated alleles were identified by PCR screening of purified genomic DNA (QIAGEN) with specific primers designed by the Jackson Laboratory (Supplemental Table 3). Adult male littermate mice were used for all studies; mice were weighed weekly and had ad libitum access to food and water.

$\mathrm{CCl}_{4}$ administration and $\mathrm{BDL}$. For induction of experimental fibrosis, mice received $200 \mu \mathrm{l}$ intraperitoneal injections twice weekly for 6 weeks with $\mathrm{CCl}_{4}(0.5 \mu \mathrm{l} / \mathrm{g}$ diluted 1:5 in olive oil; Sigma-Aldrich). Animals were sacrificed between 24 and 48 hours after the final dose. For the induction of acute $\mathrm{CCl}_{4}$ toxicity, mice received one $200-\mu 1$ intraperitoneal injection of $\mathrm{CCl}_{4}(1 \mu \mathrm{l} / \mathrm{g}$ diluted 1:5 in olive oil) 24 hours before sacrifice. Control animals received only olive oil vehicle.

For induction of cholestatic liver injury, a complete ligation of the bile duct was carried as previously described (41). Mice were maintained up to 10 days with the ligature in place (initiation and progression of fibrosis), then sacrificed for sampling.

Animal diets. Animals between 7 and 9 weeks of age were placed on high-fat feeding (60\% fat, Research Diets) for 12 and 25 weeks, or methionine-choline-deficient feeding for up to 4 weeks (MCD diet, Research Diets) with ad libitum access to feed and water. Normal chow control animals received a normal chow diet with ad libitum access to food and water.

Serology. Blood samples were collected into heparinized or EDTA-coated tubes (Sarstedt) by cardiac puncture or from the retro-orbital sinus, shortly after mice were sacrificed by cervical dislocation. Blood samples were then immediately centrifuged at $10,000 \mathrm{~g}$ for 10 minutes at $4^{\circ} \mathrm{C}$. Supernatant plasma was aliquoted and stored at $-20^{\circ} \mathrm{C}$ until analysis. Randox kits were used for measurement of plasma transaminases and triglycerides.

$q R T-P C R$. RNA was extracted from tissues or cells using the RNeasy RNA Mini Kit (QIAGEN). Complementary DNA was synthesized using M-MLV or Superscript II reverse transcriptase kits (ThermoFisher). Reactions for qRT-PCR were prepared with sequence-specific primers and the MESA green kit for SYBR green assays (Eurogentec). 18S and/or GAPDH were used as housekeeping genes for normalized relative quantification of mRNA expression using the Livak and Schmittgen $\left(2^{-\Delta \Delta C t}\right)$ method (42). Primers are listed in Supplemental Table 4.

Histology and immunohistochemistry of mouse liver. Immediately after collection, tissues were drop-fixed in $10 \%$ formalin (Sigma-Aldrich) for 24 hours, then put through an automated carousel processor for dehydration, clearing, and paraffin embedding (Leica). Liver sections were prepared at $6 \mu \mathrm{M}$ thick on polyL-lysinated slides (Sigma-Aldrich, VWR). Sections were cleared and rehydrated for all staining procedures, then dehydrated before mounting with DPX polymerizing mountant (Sigma-Aldrich). H\&E (Sigma-Aldrich) staining was used to evaluate tissue morphology; RP (Sigma-Aldrich) staining was used to visualize collagen fibers following the manufacturer's instructions. Epitope-specific antibodies were used for IHC detection of F4/80, aSMA, FasL, and IRF5 staining (Supplemental Table 5). For IHC analysis, cleared and rehydrated sections were quenched with $3 \% \mathrm{H}_{2} \mathrm{O}_{2}$ for 15 minutes at room temperature then washed twice for 5 minutes each with TBS $+0.1 \%$ (vol/vol) Tween-20. Sections were then blocked with TBS $+3 \%$ (wt/vol) BSA for 30 minutes at room temperature. Sections were then incubated with diluted primary antibodies, washed 3 times for 5 minutes with TBS $+0.1 \%$ (vol/vol) Tween-20, followed by incubation with appropriate HRP-conjugated secondary antibodies (Bio-Rad). Sections were then washed twice for 5 minutes with TBS and chromogenic detection was carried out with the DAB chromogen kit (Dako). Sections were washed in running water for 5 minutes and nuclei were counterstained with hematoxylin, followed by dehydration and mounting. Images were acquired on an Axiovert 200M microscope. Unless otherwise 


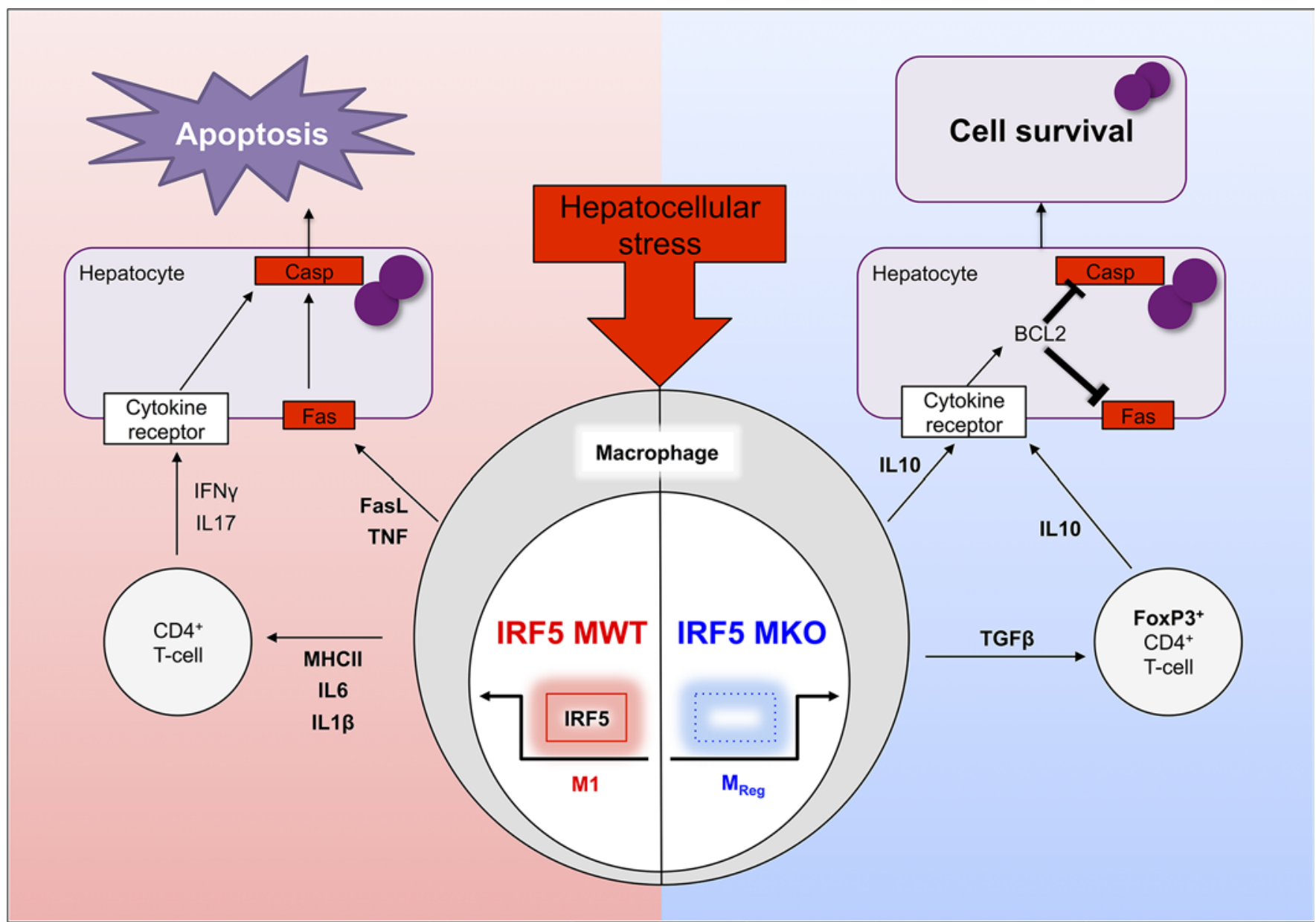

Figure 10. Schematic representation of the role of IRF5 in liver macrophages in response to hepatocellular stress. Interferon regulatory factor 5 (IRF5)-competent liver macrophages undergo proinflammatory activation in response to hepatocellular stress. Proinflammatory activation (M1) induces inflammatory cytokine and death effector release that induces hepatocyte caspase (Casp)-dependent apoptosis. Activated factors include Fas ligand (FasL), TNF, MHC II, IL6, and IL1 $\beta$. Proinflammatory activation induces type 1 and type 17 responses from CD4+ ${ }^{+}$cells. Type 1 and 17 cytokines include IL17 and IFN $\gamma$. In IRF5-deficient macrophages, hepatocellular stress leads to immunosuppressive $\left(M_{\text {Reg }}\right.$ ) polarization and secretion of IL10 and TCF $\beta$. TCF $\beta$ expression induces differentiation of $\mathrm{CD}^{+}{ }^{+} T$ cells into $\mathrm{CD}^{+}{ }^{+} \mathrm{FoxP3}^{+} \mathrm{T}$ cells, which contribute to IL10 release. Secretion of IL10 in IRF5 deficiency promotes antiapoptotic signaling in hepatocytes mediated by B cell lymphoma 2 (BCL2) family members. This process maintains cell survival under stress.

stated, IHC reagents were all sourced from Sigma-Aldrich. Quantitative expression of all immunostainings was performed using positive pixels algorithm (Indica Labs) on digital slides (Aperio). Results are expressed as percentage of positive pixels. Quantification method is an automated observer-independent process is based on whole-slide scanning and application of publicly accessible algorithms. Each biological replicate represented one slide, which was mounted with at least 3 tissue sections, representing 3 technical replicates, the mean of which was presented as the result per biological replicate.

Hepatocyte and mononuclear cell separation. Livers were washed in PBS then transferred to collagenase digestion solution in DMEM (Gibco) $\left(0.1 \mathrm{mg} / \mathrm{ml}\right.$ collagenase, $0.1 \mathrm{mg} / \mathrm{ml} \mathrm{MgCl}_{2}{ }^{*} 6 \mathrm{H}_{2} \mathrm{O}, 0.1 \mathrm{mg} / \mathrm{ml} \mathrm{CaCl}_{2}$, $0.2 \mathrm{M}$ HEPES, $200 \mathrm{U} / 1$ penicillin, $0.1 \mathrm{mg} / \mathrm{ml}$ streptomycin, $20 \mathrm{~g} / 1 \mathrm{BSA}$; Sigma-Aldrich). The liver was rapidly and thoroughly perfused with collagenase solution, then cut into small pieces and homogenized by repeated passage through a $2.5-\mathrm{ml}$ syringe. Lysate was then passed through a $100-\mu \mathrm{M}$ filter on ice, and 35 $\mathrm{ml}$ of cold HBSS $+3 \%$ (wt/vol) BSA was added. Lysate was centrifuged for 5 minutes at $40 \mathrm{~g}$; the resulting pellet composed the hepatocyte fraction, and the supernatant was composed of LMNCs. The hepatocyte pellet was resuspended in unsupplemented WEM and centrifuged for 5 minutes at $500 \mathrm{~g}$; supernatant was removed, and pellet was snap-frozen in liquid nitrogen. The LMNC fraction was centrifuged at $500 \mathrm{~g}$ for 5 minutes and resuspended in RPMI $+2 \%$ (vol/vol) fetal calf serum $+40 \%$ (vol/vol) Percoll, which was then layered onto RPMI $+2 \%$ (vol/vol) fetal calf serum $+80 \%$ (vol/vol) Percoll (Sigma-Aldrich). The 
Table 3. Physical and biochemical parameters associated with the control patients, fatty liver, NASH and HCV patients

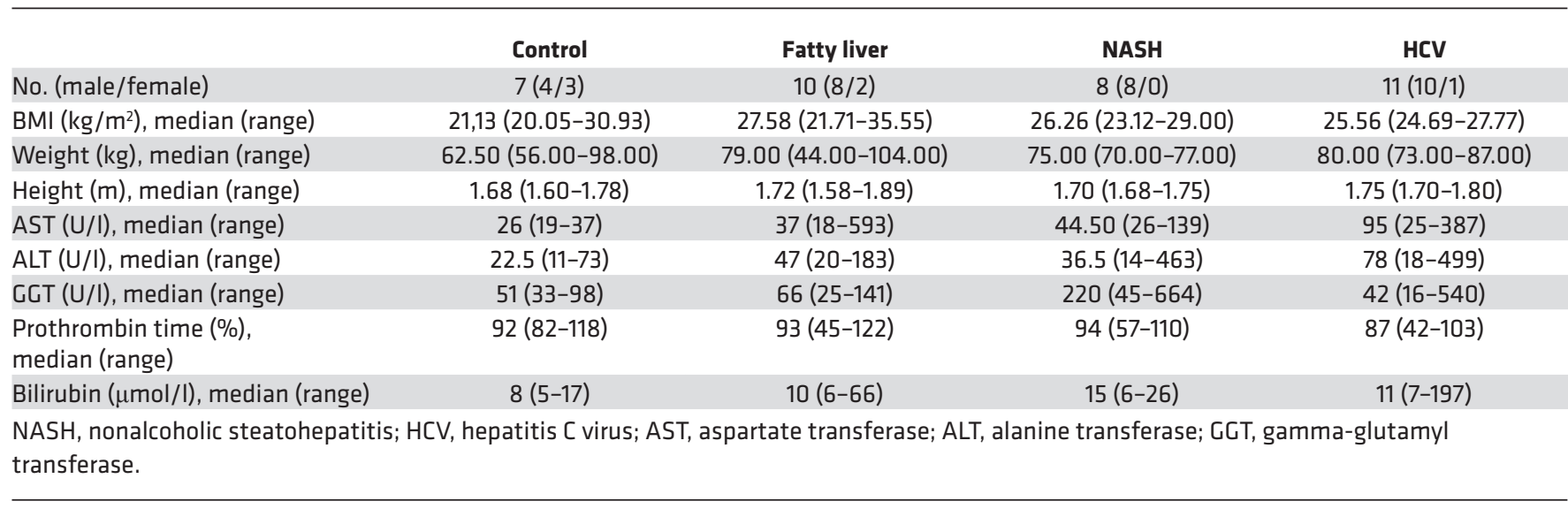

Percoll gradient was then centrifuged for 20 minutes at $800 \mathrm{~g}$ with no acceleration or deceleration. Leukocytes appear with the ring of red blood cells between gradients. Leukocyte layer was transferred to a new tube and resuspended in red blood cell lysis buffer $\left(\mathrm{NH}_{4} \mathrm{Cl} 9 \mathrm{~g} / 1, \mathrm{KHCO}_{3} 1 \mathrm{~g} / 1\right.$, EDTA $\left.37 \mathrm{mg} / 1\right)$. Samples were agitated for 3 minutes at room temperature, then centrifuged for 10 minutes at $500 \mathrm{~g}$. Resulting pellet was resuspended in flow cytometry staining buffer (HEPES $25 \mathrm{mM}$, fetal calf serum 1\% [vol/vol], EDTA $2 \mathrm{mM}$ in PBS pH 7.4). Cells were counted and redistributed according to applications.

Immunoselection of $\mathrm{F} 4 / 80^{+}$cells. At least $3 \times 10^{6}$ cells were stained for immunoselection of $\mathrm{F} 4 / 80^{+}$cells by a MACS system (Miltenyi Biotec). After isolation, cells were washed by centrifugation at $500 \mathrm{~g}$ for 5 minutes at $4^{\circ} \mathrm{C}$, then resuspended in MACS buffer (PBS + EDTA $2 \mathrm{mM}+0.2 \% \mathrm{wt} / \mathrm{vol} \mathrm{BSA}$ at $\mathrm{pH} 7.2$ ) containing the appropriate dilution of biotinylated F4/80 antibody (Miltenyi Biotec). Cells were incubated at $4^{\circ} \mathrm{C}$ in the dark for 10 minutes, then washed by centrifugation at $300 \mathrm{~g}$ for 10 minutes at $4^{\circ} \mathrm{C}$. Cells were then resuspended in MACS buffer containing the appropriate dilution of anti-biotin-conjugated magnetic microbeads (Miltenyi Biotec); staining was carried out at $4^{\circ} \mathrm{C}$ for 10 minutes in the dark; and cells were washed by centrifugation and resuspended in MACS buffer. Cell suspensions were then passed through LS columns (Miltenyi Biotec) according to the manufacturer's instructions. Both $\mathrm{F} 4 / 80^{+}$and F4/80- cell fractions were collected, washed in PBS, and snap-frozen in liquid nitrogen.

Flow cytometric analysis of LMNCs. $10^{6}$ cells and $3 \times 10^{6}$ cells were taken for surface and intracellular staining, respectively. Cells were centrifuged at $4^{\circ} \mathrm{C}$ at $500 \mathrm{~g}$ for 5 minutes and then resuspended in flow cytometry staining buffer containing fluorochrome-conjugated antibodies and stained in the dark for 30 minutes at $4^{\circ} \mathrm{C}$. Cells were then fixed and/or permeabilized according to the manufacturer's protocol of the FoxP3/transcription factor staining buffer set (Affymetrix eBioscience). For surface stains, cells were then washed by centrifugation and resuspended in flow cytometry staining buffer, then filtered at $40 \mu \mathrm{M}$ before acquisition. For nuclear stains and cytoplasmic epitopes, cells were then stained with appropriate fluorochrome-conjugated antibodies in permeabilization buffer (Affymetrix eBioscience) for 20 minutes at room temperature in the dark. Cells were then washed, resuspended in flow cytometry staining buffer, then filtered at $40 \mu \mathrm{M}$ before acquisition. LIVE/DEAD Fixable dye (Invitrogen) was used to discriminate between live and dead cells. Acquisitions were made on a BD FACSAria III (BD Biosciences).

For functional assays of cytokine expression, after isolation cells were stimulated either with $1 \mu \mathrm{g} / \mathrm{ml}$ LPS (macrophage stimulation) or $500 \mathrm{ng} / \mathrm{ml} \mathrm{PMA}$ and $500 \mathrm{ng} / \mathrm{ml}$ ionomycin (lymphocyte stimulation) in RPMI $1640+10 \%$ (vol/vol) fetal calf serum, 2 mM L-glutamine, 10 mM HEPES, 200 U/L penicillin, 0.1 $\mathrm{mg} / \mathrm{ml}$ streptomycin, $50 \mu \mathrm{M}$ 2-mercaptoethanol (Sigma-Aldrich, Gibco) in a humidified chamber with $5 \%$ $\mathrm{CO}_{2}$ at $37^{\circ} \mathrm{C}$ for 4 hours, and GolgiStop protein transport inhibitor was added immediately before use (BD Pharmingen). Cells were then blocked and washed by centrifugation before staining surface and intracellular epitopes. See Supplemental Table 6 for markers and reagents used.

$R N A$ sequencing and transcriptomic analysis. Library preparation and Illumina sequencing were performed at the Ecole normale supérieure Genomic Platform (Paris, France). Messenger (polyA ${ }^{+}$) RNAs were purified from $30 \mathrm{ng}$ of total RNA using oligo(dT). Libraries were prepared using the strand-specific RNA-Seq library 
preparation TruSeq Stranded mRNA kit (Illumina). Libraries were multiplexed by 6 on a run. A 75-bp read sequencing was performed on a NextSeq 500 device (Illumina). A mean of $38 \pm 3$ million passing Illumina quality filter reads was obtained for each of the 6 samples The analyses were performed using the Eoulsan pipeline (43), including read filtering, mapping, alignment filtering, read quantification, normalization, and differential analysis: Before mapping, poly $\mathrm{N}$ read tails were trimmed, reads $\leq 40$ bases were removed, and reads with quality mean $\leq 30$ were discarded. Reads were then aligned against the genome using STAR (version 2.4.0k) (44), and Ensembl annotation version 81 Alignments from reads matching more than once on the reference genome were removed using the Java version of SAMtools (45). To compute gene expression, GRCm38.p4 GFF3 genome annotation version 81 from the Ensembl database (http://useast.ensembl.org) was used. All overlapping regions between alignments and referenced exons were counted using HTSeqcount 0.5.3 (46). The sample counts were normalized using DESeq 1.8.3 (47). Statistical treatments and differential analyses were also performed using DESeq 1.8.3. The RNASeq gene expression data and raw fastq files are available in the GEO repository (http://www.ncbi.nlm.nih.gov/geo) under accession number GSE82157. For ontological clustering of genes, the database for annotation, visualization, and integrated discovery (DAVID) was used, and for pathway analyses the Panther classification system was used. For visualization of results, Treeview 3.0 and Cluster 3.0 applications were used (48-53).

Statistics. All data are expressed as arithmetic mean \pm SEM, unless otherwise stated. Statistical significance was evaluated by unpaired 2-tailed Students' $t$ test or by 1/2-way ANOVA with Bonferroni's post hoc test. Pearson's or Spearman's test was used to determine correlations. $P$ values less than 0.05 were considered significant. All statistical analyses were carried out using GraphPad prism 6 statistical software.

Study approval. All procedures were carried out according to the French guidelines for the care and use of experimental animals. All animal studies were approved by the regional veterinary services of the Paris police headquarters (agreement no. 75-956) and by the Biological Services Unit of the University of Paris (UPMC). All patients gave written informed consent, and the study protocol was approved by the ethics committee of Beaujon Hospital.

\section{Author contributions}

FA and NV designed research studies, analyzed data, and wrote the manuscript. FA, RB, LO, and IH conducted the in vivo experiments, and acquired and analyzed data. FL, MA, and VP conducted human analysis and wrote the manuscript. CB and SL conducted transcriptomic analyses. DGS conducted ChIPseq analysis. AL, IAU, and FF helped in the experimental design and wrote the manuscript

\section{Acknowledgments}

We are grateful to the donors for the provision of tissue samples. We would like to thank the employees of the Cellular Imaging and Cytometry Centre of the Cordeliers Research Centre, Hélène Fohrer-Ting, Estelle Devêvre, and Christophe Klein for their guidance, patience, and support. We also extend our deepest gratitude to the Biological Services Unit of the University of Paris for help with animal maintenance. We thank Tissutheque de Beaujon for providing human liver samples. We thank Tariq Khoyratty (University of Oxford) for help with ChIP-Seq figure preparation. This work was supported by grants from the French National Agency of Research (PROVIDE, to NV and AL), Region Ile de France (CORDDIM, to FA), Paris city (EMERGENCE, to NV), European Foundation for the Study of Diabetes (to NV), French Foundation for Medical Research (Equipe FRM DEQ20140329504, to NV and FF), and La Ligue contre le Cancer (to VP). RNA sequencing and transcriptome analysis were supported by the France Génomique national infrastructure, funded as part of the "Investissements d'Avenir" program managed by the Agence Nationale de la Recherche (ANR-10-INBS-09).

Address correspondence to: Fawaz Alzaid or Nicolas Venteclef, 15 Rue de l'École de Médecine, Centre de Recherche des Cordeliers, Bâtiment E, 4ème étage à gauche, Paris, 75006, France. Phone: 33.0.1.44.27.24.31; E-mail: fawaz.alzaid@inserm.fr (F. Alzaid); nicolas.venteclef@upmc.fr (N. Venteclef).

1. Diehl AM. Cytokine regulation of liver injury and repair. Immunol Rev. 2000;174:160-171.

2. Lalor PF, Shields P, Grant A, Adams DH. Recruitment of lymphocytes to the human liver. Immunol Cell Biol. 2002;80(1):52-64.

3. Wang K, Lin B, Brems JJ, Gamelli RL. Hepatic apoptosis can modulate liver fibrosis through TIMP1 pathway. Apoptosis. 2013;18(5):566-577. 
4. Malhi H, Guicciardi ME, Gores GJ. Hepatocyte death: a clear and present danger. Physiol Rev. 2010;90(3):1165-1194.

5. Davies LC, Jenkins SJ, Allen JE, Taylor PR. Tissue-resident macrophages. Nat Immunol. 2013;14(10):986-995.

6. Duffield JS, et al. Selective depletion of macrophages reveals distinct, opposing roles during liver injury and repair. J Clin Invest. 2005; 115(1):56-65.

7. Sato A, et al. Involvement of the TNF and FasL produced by CD11b Kupffer cells/macrophages in CCl4-induced acute hepatic injury. PLoS One. 2014;9(3):e92515.

8. Krausgruber T, Saliba D, Ryzhakov G, Lanfrancotti A, Blazek K, Udalova IA. IRF5 is required for late-phase TNF secretion by human dendritic cells. Blood. 2010;115(22):4421-4430.

9. Eum HA, Billiar TR. TNF/TNF receptor 1-mediated apoptosis in hepatocytes. Adv Exp Med Biol. 2011;691:617-624.

10. Lech M, Anders HJ. Macrophages and fibrosis: how resident and infiltrating mononuclear phagocytes orchestrate all phases of tissue injury and repair. Biochim Biophys Acta. 2013;1832(7):989-997.

11. Sica A, Mantovani A. Macrophage plasticity and polarization: in vivo veritas. J Clin Invest. 2012;122(3):787-795.

12. Günthner R, Anders HJ. Interferon-regulatory factors determine macrophage phenotype polarization. Mediators Inflamm. 2013;2013:731023.

13. Courties G, et al. In vivo silencing of the transcription factor IRF5 reprograms the macrophage phenotype and improves infarct healing. J Am Coll Cardiol. 2014;63(15):1556-1566.

14. Weiss M, et al. IRF5 controls both acute and chronic inflammation. Proc Natl Acad Sci U S A. 2015;112(35):11001-11006.

15. Weiss M, et al. IRF5 controls both acute and chronic inflammation. Proc Natl Acad Sci U S A. 2015;112(35):11001-11006.

16. Dalmas E, et al. Irf5 deficiency in macrophages promotes beneficial adipose tissue expansion and insulin sensitivity during obesity. Nat Med. 2015;21(6):610-618.

17. Weiss M, Blazek K, Byrne AJ, Perocheau DP, Udalova IA. IRF5 is a specific marker of inflammatory macrophages in vivo. Mediators Inflamm. 2013;2013:245804.

18. Krausgruber T, et al. IRF5 promotes inflammatory macrophage polarization and TH1-TH17 responses. Nat Immunol. 2011;12(3):231-238.

19. Saliba DG, et al. IRF5:RelA interaction targets inflammatory genes in macrophages. Cell Rep. 2014;8(5):1308-1317.

20. Friedman SL. Seminars in medicine of the Beth Israel Hospital, Boston. The cellular basis of hepatic fibrosis. Mechanisms and treatment strategies. N Engl J Med. 1993;328(25):1828-1835.

21. Epidemiology, clinical presentation laboratory investigation of severe acute respiratory syndrome (SARS) in Canada, March 2003. Can Commun Dis Rep. 2003;29(8):71-75.

22. Krausgruber T, et al. IRF5 promotes inflammatory macrophage polarization and TH1-TH17 responses. Nat Immunol. 2011;12(3):231-238.

23. Oh da Y, Olefsky JM. G protein-coupled receptors as targets for anti-diabetic therapeutics. Nat Rev Drug Discov. 2016;15(3):161-172.

24. Yang L, Feng D, Bi X, Stone RC, Barnes BJ. Monocytes from Irf5-/- mice have an intrinsic defect in their response to pristane-induced lupus. J Immunol. 2012;189(7):3741-3750.

25. Herbert DR, Orekov T, Perkins C, Finkelman FD. IL-10 and TGF-beta redundantly protect against severe liver injury and mortality during acute schistosomiasis. J Immunol. 2008;181(10):7214-7220.

26. Weiskirchen R, Tacke F. Cellular and molecular functions of hepatic stellate cells in inflammatory responses and liver immunology. Hepatobiliary Surg Nutr. 2014;3(6):344-363.

27. Fabregat I, et al. TGF- $\beta$ signalling and liver disease. FEBS J. 2016;283(12):2219-2232.

28. Wu D, et al. Eosinophils sustain adipose alternatively activated macrophages associated with glucose homeostasis. Science. 2011;332(6026):243-247.

29. Qiu Y, et al. Eosinophils and type 2 cytokine signaling in macrophages orchestrate development of functional beige fat. Cell. 2014;157(6):1292-1308.

30. Heredia JE, et al. Type 2 innate signals stimulate fibro/adipogenic progenitors to facilitate muscle regeneration. Cell. 2013;153(2):376-388

31. Hammam O, et al. The role of fas/fas ligand system in the pathogenesis of liver cirrhosis and hepatocellular carcinoma. Hepat Mon. 2012;12(11):e6132.

32. Alkhouri N, Carter-Kent C, Feldstein AE. Apoptosis in nonalcoholic fatty liver disease: diagnostic and therapeutic implications Expert Rev Gastroenterol Hepatol. 2011;5(2):201-212.

33. Ibrahim SH, Kohli R, Gores GJ. Mechanisms of lipotoxicity in NAFLD and clinical implications. J Pediatr Gastroenterol Nutr. 2011;53(2):131-140.

34. Weber-Nordt RM, et al. Interleukin-10 increases Bcl-2 expression and survival in primary human CD34+ hematopoietic progenitor cells. Blood. 1996;88(7):2549-2558.

35. Claudio PP. Immunohistochemical investigation of new suppressor oncogene p130 in oral squamous cell carcinoma, vol.35 (1999), 321-325. Oral Oncol. 2000;36(5):497.

36. Thompson K, Maltby J, Fallowfield J, McAulay M, Millward-Sadler H, Sheron N. Interleukin-10 expression and function in experimental murine liver inflammation and fibrosis. Hepatology. 1998;28(6):1597-1606.

37. de la Coste A, et al. Differential protective effects of Bcl-xL and Bcl-2 on apoptotic liver injury in transgenic mice. Am J Physiol. 1999;277(3 Pt 1):G702-G708.

38. Couzinet A, et al. A cell-type-specific requirement for IFN regulatory factor 5 (IRF5) in Fas-induced apoptosis. Proc Natl Acad Sci USA. 2008;105(7):2556-2561.

39. Bedossa $\mathrm{P}$, et al. Histopathological algorithm and scoring system for evaluation of liver lesions in morbidly obese patients. Hepatology. 2012;56(5):1751-1759.

40. Goodman ZD. Grading and staging systems for inflammation and fibrosis in chronic liver diseases. J Hepatol. 2007;47(4):598-607.

41. Tag CG, et al. Bile duct ligation in mice: induction of inflammatory liver injury and fibrosis by obstructive cholestasis. $J$ Vis Exp. $2015 ;(96)$.

42. Livak KJ, Schmittgen TD. Analysis of relative gene expression data using real-time quantitative PCR and the 2(-Delta Delta C(T)) method. Methods. 2001;25(4):402-408. 
43. Jourdren L, Bernard M, Dillies MA, Le Crom S. Eoulsan: a cloud computing-based framework facilitating high throughput sequencing analyses. Bioinformatics. 2012;28(11):1542-1543.

44. Dobin A, et al. STAR: ultrafast universal RNA-seq aligner. Bioinformatics. 2013;29(1):15-21.

45. Li H, et al. The Sequence Alignment/Map format and SAMtools. Bioinformatics. 2009;25(16):2078-2079.

46. Anders S, Pyl PT, Huber W. HTSeq - a Python framework to work with high-throughput sequencing data. Bioinformatics. 2015;31(2):166-169

47. Anders S, Huber W. Differential expression analysis for sequence count data. Genome Biol. 2010;11(10):R106.

48. Huang DW, et al. DAVID bioinformatics resources: expanded annotation database and novel algorithms to better extract biology from large gene lists. Nucleic Acids Res. 2007;35(Web Server issue):W169-W175.

49. Mi H, Poudel S, Muruganujan A, Casagrande JT, Thomas PD. PANTHER version 10: expanded protein families and functions, and analysis tools. Nucleic Acids Res. 2016;44(D1):D336-D342.

50. Mi H, Thomas P. PANTHER pathway: an ontology-based pathway database coupled with data analysis tools. Methods Mol Biol. 2009;563:123-140.

51. Huang da W, Sherman BT, Lempicki RA. Bioinformatics enrichment tools: paths toward the comprehensive functional analysis of large gene lists. Nucleic Acids Res. 2009;37(1):1-13.

52. Saldanha AJ. Java Treeview — extensible visualization of microarray data. Bioinformatics. 2004;20(17):3246-3248

53. Eisen MB, Spellman PT, Brown PO, Botstein D. Cluster analysis and display of genome-wide expression patterns. Proc Natl Acad Sci USA. 1998;95(25):14863-14868. 